\title{
THE NATURE OF GENERALIZED SCALES
}

\author{
NICOLAI STAMMEIER
}

\begin{abstract}
The notion of a generalized scale emerged in recent joint work with AfsarBrownlowe-Larsen on equilibrium states on $C^{*}$-algebras of right LCM monoids, where it features as the key datum for the dynamics under investigation. This work provides the structure theory for such monoidal homomorphisms. We establish uniqueness of the generalized scale and characterize its existence in terms of a simplicial graph arising from a new notion of irreducibility inside right LCM monoids. In addition, the method yields an explicit construction of the generalized scale if existent. We discuss applications for graph products as well as algebraic dynamical systems, and reveal a striking connection to Saito's degree map.
\end{abstract}

\section{INTRODUCTION}

The notion of a generalized scale first appeared in [ABLS17] as an ad-hoc definition that allowed for an abstract and unified theory of equilibrium states for a number of quantum statistical mechanical systems [LR10, BaHLR12, LRR11, LRRW14, CaHR16]. More precisely, all these case studies can be viewed as considerations for $C^{*}$-algebras of right LCM monoids, that is, left cancellative monoids in which the intersection of two principal right ideals is either empty or another principal right ideal again. Here, right LCM refers to the existence of a right Least Common Multiple given the presence of a right common multiple. We note that right LCM monoids are commonly referred to as right LCM semigroups in the operator algebraic literature as the presence of a unit is taken for granted in this context. Since this work is part of semigroup theory rather than operator algebras, we decided to correct this potentially misleading terminology.

In order to give the definition of a generalized scale, it is convenient to first recall some notation: Let $S$ be a right LCM monoid. For $s, t \in S$, we follow the notation of [Spi12] and write $s \cap t$ if $s S \cap t S$ is nonempty, and $s \perp t$ otherwise, in which case we call $s$ and $t$ orthogonal. An important submonoid of a right LCM monoid $S$ is its core $S_{c}:=\{a \in S \mid a$ ก $s$ for all $s \in S\}$ introduced in [Star15], inspired by [CL07]. As in [ABLS17, Subsection 2.1], $s, t \in S$ are core equivalent, denoted $s \sim t$, if there are $a, b \in S_{c}$ with $s a=t b$. It will be convenient to work with the following definition of a generalized scale that avoids the notion of accurate foundation sets:

Definition 1.1. A generalized scale on a right LCM monoid $S$ is a nontrivial homomorphism $N: S \rightarrow \mathbb{N}^{\times}, s \mapsto N_{s}$ satisfying

(i) $\left|N^{-1}(n) / \sim\right|=n$ for all $n \in N(S)$;

Date: March 8, 2019.

2010 Mathematics Subject Classification. $20 \mathrm{M} 15$.

Key words and phrases. right LCM monoids, growth functions, Saito's degree maps, graph products, $\mathrm{C}^{*}$-algebras, KMS-states.

This research was supported by RCN through FRIPRO 240362. 
(ii) $N_{s}=N_{t}$ implies $s \sim t$ or $s \perp t$; and

(iii) for all $s \in S, n \in N(S)$, there exists $t \in N^{-1}(n)$ with $s$ ก $t$.

Proposition 2.5 will establish that this definition is equivalent to the original one given in [ABLS17, Definition 3.1(A3)]. The assumption that $N$ has to be nontrivial excludes precisely the left reversible right LCM monoids and is needed to obtain a nontrivial time evolution of the quantum statistical mechanical system based on $C^{*}(S)$, see [ABLS17, Section 4].

Arguably, the definition of a generalized scale was useful, but rather elusive. It is thus natural to ask basic questions such as:

1. How many generalized scales may a right LCM monoid have?

2. When does a right LCM monoid admit a generalized scale?

3. Which structural aspects are captured by a generalized scale?

Already in [Sta18, Corollary 4.9] it had been observed that right-angled Artin monoids have at most one generalized scale. These monoids were first considered in [CF69] (also known as free partially commutative monoids or trace monoids). This result can then be further exploited with the help of [ABLS17, Proposition 5.4] to show uniqueness of the generalized scale for self-similar group actions [ABLS17, Subsection 5.3] and BaumslagSolitar monoids with positive parameters [ABLS17, Subsection 5.5]. Combining [Sta18, Corollary 4.5] with [ABLS17, Proposition 3.6(v)], it follows that there is an abundance of right LCM monoids without generalized scales.

In the present work, we start by answering the first question by establishing a uniqueness theorem for generalized scales, see Theorem 3.6. Its proof relies on the preservation of arithmetic aspects of $\mathbb{N}^{\times}$under generalized scales. On the way to this result, we show that condition (A4) from [ABLS17, Definiton 3.1] is redundant, see Proposition 3.1. This is of particular relevance to [BLRSt], where, using this work, we obtain a classification of KMS-states for arbitrary right LCM monoids that admit a (necessarily unique) generalized scale. That is to say, the only requirement left for obtaining the conclusions of [ABLS17, Theorem 4.3] is the existence of a generalized scale. The guiding theme for this improvement is to work with the quotient space $S / \sim$ and the action $\alpha: S_{c} \curvearrowright S / \sim$ induced by left multiplication instead of using arithmetic structures inside the semigroup. In return, [BLRSt] indicates that a very natural direction for further progress on KMS-states in the context of semigroup $\mathrm{C}^{*}$-algebras is a sophisticated understanding of generalized scales on right LCM monoids, which is the aim of the present work.

The answers to the second and third questions are given in Theorem 4.13 and require more work: A central object in the proof of Theorem 3.6 is the set of irreducible elements $\operatorname{Irr} N(S)$ of the submonoid $N(S) \subset \mathbb{N}^{\times}$. In Proposition 4.1, we note that their preimages under $N$ are precisely the elements $s \in S$ with the following property: whenever $s=t r$ for $t, r \in S$, then either $t \in S_{c}$ or $r \in S_{c}$. We shall call these elements noncore irreducible, see Definition 4.2 , and denote their collection by $\mathcal{I}(S)$. For a basic understanding of their behavior, we keep track of whether two noncore irreducible elements $s$ and $t$ satisfy $s \perp t$ or $s \cap t$. Since this is a property of $[s],[t] \in S / \sim$ rather than their representatives, we reduce to equivalence classes. By drawing an edge between $[s]$ and $[t]$ whenever $s \cap t$, we obtain a simplicial graph $\Gamma(S)$ in Definition 4.4, the core graph of $S$. It turns out that the restriction of $\alpha$ to $\mathcal{I}(S) / \sim$ induces an action $\beta: S_{c} \curvearrowright \Gamma(S)$ by graph automorphisms, see Proposition 4.7, which may be of independent interest. The existence of a generalized 
scale implies that this action is simply a composition of permutations of the vertex sets of the coconnected components of $\Gamma(S)$, see Corollary 4.8 and Theorem 4.13 (i).

With the core graph at our disposal, we set out to characterize the existence of the generalized scale and to provide an explicit construction in Theorem 4.13. We find that, in addition to quite restrictive features of the core graph, see Theorem 4.13(i),(ii), the existence of a generalized scale also requires that

(iii) $S$ is noncore factorable, that is, every element in $S \backslash S_{c}$ be core equivalent to a finite product of noncore irreducibles; and that

(iv) $S$ has balanced factorization: for $s, t \in \mathcal{I}(S)$ with $s \cap t$, there are $s^{\prime}, t^{\prime} \in \mathcal{I}(S)$ such that $s S \cap t S=s t^{\prime} S, s t^{\prime}=t s^{\prime}$ with $\left[s^{\prime}\right]$ and $\left[t^{\prime}\right]$ belonging to the same coconnected component of $\Gamma(S)$ as $[s]$ and $[t]$, respectively.

As to be expected, showing that the conditions (i)-(iv) are not only necessary, but in fact sufficient for the existence of a generalized scale is the hard part of the proof. One of the key tools in the proof is an algorithm for computing a right LCM of products of noncore irreducibles, see Remark 4.11.

In the final section, we first describe the core graph and its features for the two elementary examples of self-similar group actions in 5.1 and the ax + b-semigroup over the natural numbers in 5.2. For algebraic dynamical systems, that is, suitable actions of right LCM monoids on discrete groups by injective group endomorphisms, the application of Theorem 4.13 is less straightforward. We examine the sufficient criterion for the existence of a generalized scale from [ABLS17, Proposition 5.11(i)], and find that it is not necessary in general, see Example 5.2. On the other hand, Ledrappier's shift is an example that appears to lack a generalized scale precisely for the violation of condition (b) of Remark 5.1, see Example 5.3. Algebraic dynamical systems also provide the flexibility to build examples where $\beta$ switches coconnected components of $\Gamma(S)$, see Example 5.4.

Motivated by the findings in [Sta18], we investigate applications to graph products of right LCM monoids, and obtain a result which reduces the considerations to the coconnected case, see Theorem 5.5. However, already Example 5.7 shows that graph products of coconnected graphs may well behave counterintuitively, depending on the combination of edge set and family of vertex semigroups. Roughly speaking, very few graph products admit generalized scales. This even remains true for the most elementary examples of this type, namely right-angled Artin monoids, see [Sta18, Corollary 4.9].

In summary, the present work shows that the generalized scale represents an intriguing set of characteristic features of the right LCM monoid in question, see Theorem 4.13. These features are common for many types of right LCM monoids, see [ABLS17]. However, there are also many interesting examples where different parts of the requirements for the existence of the generalized scale are not met. This motivates the search for a more flexible notion of such maps, the outcome of which we present in Subsection 5.5: Generalized scales correspond to special kinds of Saito's degree maps, see [Sai13] for details.

Acknowledgements: The author is grateful to Nathan Brownlowe, Nadia S. Larsen, Jacqui Ramagge, and Jack Spielberg for valuable discussions. He wishes to thank the anonymous referee for valuable comments leading to an improved exposition of the material, in particular for suggesting an alternative proof to Proposition 3.1, see Remark 3.2, 
and for spotting details in the proofs of Lemma 4.12 (i) and Theorem 4.13 that needed extra care.

\section{Preliminaries}

In an attempt to make this work essentially self-contained, we shall briefly recall the auxiliary results from [ABLS17] that we will need and make a few additional observations to help the reader familiarize with the concepts of this work. Throughout, let $S$ be a right LCM monoid. Let us start with the first part of [ABLS17, Lemma 3.5]:

Lemma 2.1. Let $a \in S_{c}$ and $s \in S$. If $b \in S$ satisfies $a S \cap s S=s b S$, then $b \in S_{c}$.

Proof. Let $a \in S_{c}$ and $s, b \in S$ satisfy $s S \cap a S=s b S$ and fix $t \in S$. Then

$$
s(t S \cap b S)=s t S \cap s S \cap a S=s t S \cap a S \neq \emptyset
$$

shows $t \cap b$, and hence $b \in S_{c}$ since $t$ was arbitrary.

Recall that a subsemigroup $T \subset S$ is said to be hereditary if it has the following property: If $s \in S$ satisfies $t \in s S(t \geq s)$ for some $t \in T$, then $s \in T$.

Lemma 2.2. The core $S_{c}$ is a hereditary submonoid of $S$. In particular, if $s, t \in S$ satisfy $s \sim t$, then $s S \cap t S=$ saS for some $a \in S_{c}$.

Proof. For the first claim, we note that for $a \in S, b \in S_{c}$ with $b \in a S$, we have $a S \cap s S \supset$ $b S \cap s S \neq \emptyset$ for all $s \in S$, so that $a \in S_{c}$. For the second part, $s \sim t$ implies that there are $b, c \in S_{c}$ with $s b=t c$. So we have $s S \cap t S=s a S$ for some $a \in S$ with $b \in a S$. Thus the first part yields $a \in S_{c}$.

Lemma 2.3. Let $s, t \in S$. Then $s \sim t$ holds if and only if $s \cap r \Leftrightarrow t \cap r$ for all $r \in S$.

Proof. Let $s, t \in S$. Suppose first that $s \sim t$. Lemma 2.2 shows that $s S \cap t S=s a S$ with $s a=t b$ for some $a, b \in S_{c}$. For $r \in S$, we thus get $r \cap s \Leftrightarrow r \cap s a=t b \Leftrightarrow r \cap t$.

Conversely, assume that $s \cap r \Leftrightarrow t \cap r$ for all $r \in S$. In particular, picking $r:=s$ yields $s$ ก $t$, say $s S \cap t S=s a S$ with $s a=t b$ for some $a, b \in S$. We claim that $a$ and $b$ belong to $S_{c}$. For $p \in S$, let us consider $s p$. As $s p \cap s$, we have $s p \cap t$, which allows us to conclude

$$
s(p S \cap a S)=s p S \cap s S \cap t S=s p S \cap t S \neq \emptyset .
$$

Hence we obtain $a$ ก $p$ for all $p \in S$, which is $a \in S_{c}$. Arguing in the same way for $b$, we also get $b \in S_{c}$.

Following the terminology of [SY10], the notion of a foundation set was introduced in [BRRW14] for right LCM monoids: A foundation set is a finite subset $F \subset S$ (we write $F \subset S$ to indicate this) such that for every $s \in S$ there is $t \in F$ with $s \cap t$. This was refined in [BS16] to the notion of an accurate foundation set, which is a foundation set $F$ satisfying $s \perp t$ for all $s, t \in F, s \neq t$.

According to [ABLS17, Definition 3.1(A3)], a nontrivial homomorphism $N: S \rightarrow \mathbb{N}^{\times}$ is called a generalized scale if

(a) $\left|N^{-1}(n) / \sim\right|=n$ for all $n \in N(S)$; and

(b) for each $n \in N(S)$, every transversal for $N^{-1}(n) / \sim$ is an accurate foundation set for $S$. 
Let us recall some key properties for generalized scales, namely [ABLS17, Proposition 3.6(i)-(iv)] without proof.

Proposition 2.4. Let $N: S \rightarrow \mathbb{N}^{\times}$be a generalized scale in the sense of [ABLS17, Definition 3.6(A3)].

(i) $N^{-1}(1)=S_{c}$, and this is a proper subsemigroup of $S$.

(ii) For $s, t \in S$ with $N_{s}=N_{t}$, either $s \sim t$ or $s \perp t$ holds.

(iii) Let $F$ be a foundation set for $S$ with $F \subset N^{-1}(n)$ for some $n \in N(S)$. If $|F|=n$ or $F$ is accurate, then $F$ is a transversal for $N^{-1}(n) /$.

(iv) Whenever $s, t \in S$ satisfy $s S \cap t S=r S$, then $N_{s} N(S) \cap N_{t} N(S)=N_{r} N(S)$.

In particular, part (i) implies that core equivalent elements have the same image under $N$. Let us now show that the two competing notions for a generalized scale coincide:

Proposition 2.5. A nontrivial homomorphism $N: S \rightarrow \mathbb{N}^{\times}$is a generalized scale in the sense of [ABLS17, Definition 3.6(A3)] if and only if it satisfies (i)-(iii) of Definition 1.1.

Proof. As (a) and (i) are identical, we need to show that (b) is equivalent to (ii) and (iii), given (i). We observe that (b) implies (iii) by the definition of a foundation set. Proposition 2.4(ii) establishes (ii).

Conversely, suppose (i)-(iii) of Definition 1.1 hold. We need to prove (b), so let $n \in N(S)$ and fix a transversal $F$ for $N^{-1}(n) / \sim$. The set $F$ is finite by (i) and its elements are mutually orthogonal by (ii). Thus the claim reduces to showing that $F$ is a foundation set. For every $s \in S$, (iii) states that there is $t \in N^{-1}(n)$ with $s \cap t$. If $t^{\prime} \in F$ is the (unique) element satisfying $t^{\prime} \sim t$, then Lemma 2.3 shows $s \cap t^{\prime}$. Thus $F$ is an accurate foundation set, that is, (b) holds.

Corollary 2.6. For every generalized scale $N$, its image $N(S)$ is a (right) LCM subsemigroup of $\mathbb{N}^{\times}$.

Proof. Let $m, n \in N(S)$. So there is $s \in N^{-1}(m)$, and Definition 1.1 (iii) asserts that there is $t \in N^{-1}(n)$ with $s$ ก $t$, that is, $s S \cap t S=r S$ for some $r \in S$. Using Proposition 2.4 (iv), we thus get $m N(S) \cap n N(S)=N_{r} N(S)$, so that $N_{r}$ is the (unique right) LCM of $m$ and $n$.

Finally, let us recall the part of [ABLS17, Lemma 3.9] that holds without assumptions on core irreducible elements in $S$, along with its proof.

Lemma 2.7. Let $S$ be a right LCM monoid. Then left multiplication defines an action $\alpha: S_{c} \curvearrowright S / \sim$ by bijections $\alpha_{a}([s])=[a s]$. Every generalized scale $N$ on $S$ is invariant under this action.

Proof. For every $a \in S_{c}$, the map $\alpha_{a}$ is well-defined as left multiplication preserves the core equivalence relation. If as $\sim$ at for some $s, t \in S$, then $s \sim t$ by left cancellation. Hence $\alpha_{a}$ is injective. On the other hand, Lemma 2.1 states that for $s \in S$ we have $a S \cap s S=a t S, a t=s b$ for some $b \in S_{c}$ and $t \in S$. Thus $\alpha_{a}([t])=[a t]=[s]$, and we conclude that $\alpha_{a}$ is a bijection. Generalized scales are invariant under this action by Proposition 2.4 (i). 


\section{UNIQUENESS OF THE GENERALIZED SCALE}

Let us start with a few observations that will streamline the proof of the uniqueness theorem. For an LCM subsemigroup $\mathcal{N}$ of $\mathbb{N}^{\times}$, let us denote its irreducible elements by

$$
\operatorname{Irr} \mathcal{N}:=\{n \in \mathcal{N} \backslash\{1\} \mid n=k \ell \text { for } k, \ell \in \mathcal{N} \Rightarrow k=1 \text { or } \ell=1\} .
$$

Proposition 3.1. Every LCM subsemigroup $\mathcal{N}$ of $\mathbb{N}^{\times}$is freely generated by its irreducible elements. In particular, $N(S)$ is freely generated by $\operatorname{Irr} N(S)$ for every generalized scale $N: S \rightarrow \mathbb{N}^{\times}$, that is, condition (A3) of [ABLS17, Definition 3.1] implies (A4).

Proof. First, we claim that $m \mathcal{N} \cap n \mathcal{N}=m n \mathcal{N}$ whenever $m, n \in \operatorname{Irr} \mathcal{N}$ are distinct. There are unique $k, \ell \in \mathcal{N}$ with $m \mathcal{N} \cap n \mathcal{N}=m k \mathcal{N}$ and $n=k \ell$ because $m n \in m k \mathcal{N}$. As $m \neq n$ forces $k>1$ and $\mathcal{N}$ is cancellative, irreducibility of $n$ forces $\ell=1$, that is, $k=n$.

For the general case suppose to the contrary that there were $k, \ell \geq 1$ and

$$
m_{1}, \ldots, m_{k}, n_{1}, \ldots, n_{\ell} \in \operatorname{Irr} \mathcal{N} \text { with } m_{1} \cdots m_{k}=n_{1} \cdots n_{\ell},
$$

but there did not exist a bijection $f:\{1, \ldots, k\} \rightarrow\{1, \ldots, \ell\}$ such that $m_{f(j)}=n_{j}$ for all $1 \leq j \leq k$. By counting multiplicities of the elements in $\left\{m_{j} \mid 1 \leq j \leq k\right\} \cap\left\{n_{j} \mid\right.$ $1 \leq j \leq \ell\}$, we can choose subsets $K \subset\{1, \ldots, k\}$ and $L \subset\{1, \ldots, \ell\}$ and build a bijection $f^{\prime}: K \rightarrow L$ with $m_{f(j)}=n_{j}$ for all $j \in K$. By choosing $K$ maximal with the above property and removing it from $\{1, \ldots, k\}$ (and the corresponding $L$ from $\{1, \ldots, \ell\})$, the problem reduces to the following situation: There exist $k^{\prime}, \ell^{\prime} \geq 1(k=\ell$ and $K=\{1, \ldots, k\}$ cannot occur) and

$$
m_{1}, \ldots, m_{k^{\prime}}, n_{1}, \ldots, n_{\ell^{\prime}} \in \operatorname{Irr} \mathcal{N} \text { with } m_{1} \cdots m_{k^{\prime}}=n_{1} \cdots n_{\ell^{\prime}} \text { and } m_{i} \neq n_{j} \text { for all } i, j \text {. }
$$

In view of the first part of the proof, $n_{1} \neq m_{j}$ for all $j$ gives

$$
\begin{aligned}
n_{1} \mathcal{N} \cap m_{1} \cdots m_{k^{\prime}} \mathcal{N} & =n_{1} \mathcal{N} \cap m_{1} \mathcal{N} \cap m_{1} \cdots m_{k^{\prime}} \mathcal{N} \\
& =m_{1} n_{1} \mathcal{N} \cap m_{1} m_{2} \mathcal{N} \cap m_{1} \cdots m_{k^{\prime}} \mathcal{N} \\
& =m_{1}\left(n_{1} \mathcal{N} \cap m_{2} \mathcal{N}\right) \cap m_{1} \cdots m_{k^{\prime}} \mathcal{N} \\
& \vdots \\
& =m_{1} \cdots m_{k^{\prime}} n_{1} \mathcal{N} .
\end{aligned}
$$

As $n_{1}>1$, this contradicts

$$
n_{1} \mathcal{N} \cap m_{1} \cdots m_{k^{\prime}} \mathcal{N} \supset n_{1} \cdots n_{\ell^{\prime}} \mathcal{N} \cap m_{1} \cdots m_{k^{\prime}} \mathcal{N}=m_{1} \cdots m_{k^{\prime}} \mathcal{N}
$$

Hence we conclude that $\operatorname{Irr} \mathcal{N}$ freely generates a submonoid $M$ of $\mathcal{N}$. To see that $M=\mathcal{N}$, we show by induction on $n \in \mathcal{N}$ that every element in $\mathbb{N}$ factors into a finite product of elements from Irr $\mathcal{N}$ : Suppose $\mathcal{N} \neq\{1\}$ and pick the smallest $n \in \mathcal{N} \backslash\{1\}$ with respect to the total order $\leq_{\mathbb{N}}$ coming from the canonical inclusion $\mathcal{N} \subset \mathbb{N}$. Then $n \in \operatorname{Irr} \mathcal{N}$ as $n=m m^{\prime}$ forces $m, m^{\prime} \in\{1, n\}$. Next, assume that a factorization with respect to $\operatorname{Irr} \mathcal{N}$ exists for all $m \in \mathcal{N}, m \leq_{\mathbb{N}} n$ for an arbitrary but fixed $n \in \mathcal{N}$. Let $n^{\prime} \in \mathcal{N}$ be the smallest number with $n^{\prime}>_{\mathbb{N}} n$. If $n^{\prime} \in \operatorname{Irr} \mathcal{N}$, then there is nothing to prove. On the other hand, if $n^{\prime}=m m^{\prime}$ for some $m, m^{\prime} \in \mathcal{N} \backslash\{1\}$, then $m, m^{\prime} \leq_{\mathbb{N}} n$ since $n^{\prime}$ was chosen to be minimal with $n^{\prime}>_{\mathbb{N}} n$. Therefore, both $m$ and $m^{\prime}$ admit a factorization with respect to $\operatorname{Irr} \mathcal{N}$, and the product of these is a factorization for $n^{\prime}$. This completes the induction and we conclude that $\mathcal{N}$ is freely generated by $\operatorname{Irr} \mathcal{N}$. 
The claim concerning a generalized scale $N$ now follows from Corollary 2.6.

Remark 3.2. The following elegant alternative for the proof of Proposition 3.1 was suggested by the anonymous referee: It is known that an abelian cancellative monoid is free if and only if it

(a) has trivial group of units;

(b) satisfies the ascending chain condition on principal ideals; and

(c) each irreducible element is prime.

In the present case of an LCM subsemigroup $\mathcal{N} \subset \mathbb{N}^{\times}$, (a) and (b) are inherited from $\mathbb{N}^{\times}$. These two conditions imply that every element admits a factorization into a finite product of irreducibles.

In order to establish (c), it suffices to show that if $p \in \operatorname{Irr} \mathcal{N}$ and $q_{1}^{m_{1}} \cdots q_{k}^{m_{k}} \in p \mathcal{N}$ for some mutually distinct $q_{i} \in \operatorname{Irr} \mathcal{N}$ and $m_{i} \geq 1$ for all $i$, then $q_{i} \in p \mathcal{N}$ for some $i$, that is, $p=q_{i}$ as (a) holds and $q_{i}$ is irreducible. Suppose that $p \neq q_{i}$ for all $i$ and that $k$ and $\sum_{1 \leq i \leq k} m_{i}$ are minimal, that is, $q_{1}^{m_{1}^{\prime}} \cdots q_{\ell}^{m_{\ell}^{\prime}} \in p \mathcal{N}$ implies $\ell \geq k$ and $\sum_{1 \leq i \leq \ell} m_{i}^{\prime} \geq \sum_{1 \leq i \leq k} m_{i}$. Combining the first claim in the proof of Proposition 3.1 with left cancellation, we obtain

$$
\begin{aligned}
p \mathcal{N} \cap q_{1} \mathcal{N} \cap \ldots \cap q_{k} \mathcal{N} & =p q_{1} \mathcal{N} \cap p q_{2} \mathcal{N} \cap \ldots \cap p q_{k} \mathcal{N} \\
& =p\left(q_{1} \mathcal{N} \cap q_{\mathcal{N}} \cap \ldots \cap q_{k} \mathcal{N}\right) \\
& \vdots \\
& =p q_{1} q_{2} \cdots q_{k} \mathcal{N} .
\end{aligned}
$$

Thus we get

$$
q_{1}^{m_{1}} \cdots q_{k}^{m_{k}} \in p \mathcal{N} \cap q_{1} \mathcal{N} \cap \ldots \cap q_{k} \mathcal{N}=p q_{1} q_{2} \cdots q_{k} \mathcal{N},
$$

which implies $q_{1}^{m_{1}-1} \cdots q_{k}^{m_{k}-1} \in p \mathcal{N}$ by left cancellation. This contradicts minimality of $\left(m_{1}, \ldots, m_{k}\right)$.

Lemma 3.3. Let $S$ be a right LCM monoid, $M, N: S \rightarrow \mathbb{N}^{\times}$be two generalized scales on $S$, and $s \in S \backslash S_{c}$. If $M_{s}$ is reducible in $M(S)$, then there are $a \in S_{c}$ and $t, r \in S \backslash S_{c}$ such that $s a=t r$. In particular, $N_{s}$ is irreducible if and only if $M_{s}$ is irreducible.

Proof. Suppose there are $k, \ell \in M(S), k, \ell>1$ such that $M_{s}=k \ell$. By Definition 1.1 (iii), there is $t \in M^{-1}(k)$ such that $t \cap s$, say $t S \cap s S=\operatorname{tr} S$ with $\operatorname{tr}=s a$ for some $r, a \in S$. Due to Proposition 2.4 (iv) and $M_{s}=M_{t} \ell$, we know that $M_{r}=\ell>1$ and $M_{a}=1$. This implies $r \in S \backslash S_{c}$ and $a \in S_{c}$, see Proposition 2.4 (i). We conclude from this that $N_{s}=N_{s a}=N_{t} N_{r}$ with $N_{t}, N_{r}>1$, so that $N_{s}$ is reducible in $N(S)$.

Recall from [BS16, Definition 1.2 and Definition 2.1] that a finite subset $\mathcal{T} \subset S$ is an accurate foundation set if its elements are mutually orthogonal and for every $s \in S$ there is $t \in \mathcal{T}$ with $s \cap t$.

Lemma 3.4. Let $N$ be a generalized scale on $S$. If $s, t \in S$ with $N_{s}, N_{t} \in \operatorname{Irr} N(S)$ satisfy $N_{s} \neq N_{t}$, then $s \cap t$.

Proof. According to Proposition 3.1, the right LCM of $N_{s}$ and $N_{t}$ in $N(S)$ is $N_{s} N_{t}$. So if $s^{\prime} \in N^{-1}\left(N_{s}\right)$ and $t^{\prime} \in N^{-1}\left(N_{t}\right)$ satisfy $s^{\prime} S \cap t^{\prime} S=r S$ for some $r \in S$, then $N_{r}=N_{s} N_{t}$ by Proposition 2.4 (iv). 
Next, take transversals $F_{r}$ for $N^{-1}\left(N_{r}\right) / \sim$ with $r \in F_{r}$ for $r=s, t$. By [ABLS17, Definition 3.1(A3)(b)], $F_{s}$ and $F_{t}$ are accurate foundation sets with $\left|F_{r}\right|=N_{r}$ for $r=s, t$. Hence, every complete minimal set of representatives $F$ for

$$
\left\{s^{\prime} S \cap t^{\prime} S \mid s^{\prime} \in F_{s}, t^{\prime} \in F_{t}\right\} \backslash\{\emptyset\}
$$

is also an accurate foundation set. By the first paragraph, we have $F \subset N^{-1}\left(N_{s} N_{t}\right)$. According to Proposition 2.4 (iii), $F$ is a transversal for $N^{-1}\left(N_{s} N_{t}\right) / \sim$, and thus $|F|=$ $N_{s} N_{t}$. In view of $\left|F_{s}\right|=N_{s}$ and $\left|F_{t}\right|=N_{t}$, this forces $s$ ก $t$.

Lemma 3.5. Let $N$ be a generalized scale on the right $L C M$ monoid $S$ and $s \in S \backslash S_{c}$. Then there are $k \geq 1$ and $s_{1}, \ldots, s_{k} \in S$ with $N_{s_{j}} \in \operatorname{Irr} N(S)$ for all $j$ satisfying $s \sim s_{1} \cdots s_{k}$.

Proof. Let $N_{s}=n_{1} \cdots n_{k}$ with $n_{i} \in \operatorname{Irr} N(S)$ for all $i$ be the (up to permutation unique) factorization into irreducibles in $N(S) \subset \mathbb{N}^{\times}$. By Definition 1.1(iii), there is $s_{1} \in N^{-1}\left(n_{1}\right)$ with $s_{1} \cap s$, say $s_{1} S \cap s S=s_{1} r_{1} S, s_{1} r_{1}=s a_{1}$ for some $a_{1}, r_{1} \in S$. Since $N_{s_{1}}$ is a divisor of $N_{s}$, Proposition 2.4 (iv) implies that $N_{r_{1}}=n_{2} \cdots n_{k}$ and $N_{a_{1}}=1$. The latter is equivalent to $a_{1} \in S_{c}$, see Proposition 2.4 (i). Thus we obtained $s_{1} \in N^{-1}\left(n_{1}\right), a_{1} \in S_{c}$, and $r_{1} \in N^{-1}\left(n_{2} \cdots n_{k}\right)$ such that

$$
s S \cap s_{1} S=s_{1} r_{1} S, s_{1} r_{1}=s a_{1} .
$$

Applying the same process to $\left(r_{1}, n_{2}\right)$ in place of $\left(s, n_{1}\right)$ yields $s_{2} \in N^{-1}\left(n_{2}\right), a_{2} \in S_{c}$, and $r_{2} \in N^{-1}\left(n_{3} \cdots n_{k}\right)$ such that $r_{1} S \cap s_{2} S=s_{2} r_{2} S, s_{2} r_{2}=r_{1} a_{2}$. After $k-1$ steps, we have obtained $s_{i} \in N^{-1}\left(n_{i}\right), a_{i} \in S_{c}$ and $r_{i} \in N^{-1}\left(\prod_{i+1 \leq j \leq k} n_{j}\right)$ for $1 \leq i \leq k-1$ with

$$
s a_{1} \cdots a_{k-1}=s_{1} \cdots s_{k-1} r_{k-1} \quad \text { with } r_{k-1} \in N^{-1}\left(n_{k}\right) .
$$

Thus, setting $s_{k}:=r_{k-1}$ gives $s \sim s_{1} \cdots s_{k}$.

Theorem 3.6. A right LCM monoid admits at most one generalized scale.

Proof. Let $S$ be a right LCM monoid and $M, N: S \rightarrow \mathbb{N}^{\times}$be two generalized scales. Fix $s \in S$ with $N_{s} \in \operatorname{Irr} N(S)$. By Lemma 3.3, we know that $M_{s} \in \operatorname{Irr} M(S)$. We claim that $N_{s}=M_{s}$. According to Definition 1.1(i), this amounts to $\left|N^{-1}\left(N_{s}\right) / \sim\right|=\left|M^{-1}\left(M_{s}\right) / \sim\right|$. By symmetry, it thus suffices to show $N^{-1}\left(N_{s}\right) / \sim \subset M^{-1}\left(M_{s}\right) / \sim$. Suppose to the contrary that there exists $t \in N^{-1}\left(N_{s}\right)$ with $t \not s$ and $M_{t} \neq M_{s}$. By Definition 1.1(ii), $N_{t}=N_{s}$ and $t \not s$ force $s \perp t$. However, Lemma 3.3 entails $M_{s}, M_{t} \in \operatorname{Irr} M(S)$ as $N_{s}=N_{t} \in \operatorname{Irr} N(S)$. Therefore, Lemma 3.4 applied to $M$ gives $s$ ก $t$, contradicting $s \perp t$. Thus we get $N^{-1}\left(N_{s}\right) / \sim \subset M^{-1}\left(M_{s}\right) / \sim$, and hence $N_{s}=M_{s}$ for every $s \in S$ with $N_{s} \in \operatorname{Irr} N(S)$.

In addition, we know that $M$ and $N$ are homomorphisms with $\left.M\right|_{S_{c}}=1=\left.N\right|_{S_{c}}$. To conclude that $N=M$, let $s \in S \backslash S_{c}$. By Lemma 3.5, there are $k \geq 1$ and $s_{1}, \ldots, s_{k} \in S$ with $N_{s_{i}} \in \operatorname{Irr} N(S)$ such that $s \sim s_{1} \cdots s_{k}$. This allows us to deduce

$$
N_{s}=N_{s_{1}} \cdots N_{s_{k}}=M_{s_{1}} \cdots M_{s_{k}}=M_{s},
$$

that is, $M=N$. 


\section{Existence AND CONSTRUCTION OF GENERALizEd SCALES}

The idea behind the final step in the proof of the uniqueness result Theorem 3.6 will serve as our starting point: If a right LCM monoid $S$ admits a generalized scale $N: S \rightarrow \mathbb{N}^{\times}$, then every element in $S \backslash S_{c}$ is a nonempty, finite product of elements in $N^{-1}(\operatorname{Irr} N(S))$. We can characterize these minimal elements in the following way:

Proposition 4.1. Let $N: S \rightarrow \mathbb{N}^{\times}$be a generalized scale. Then $s \in S \backslash S_{c}$ satisfies $N_{s} \in \operatorname{Irr} N(S)$ if and only if $s a=\operatorname{tr}$ for $a \in S_{c}$ and $t, r \in S$ implies either $t \in S_{c}$ or $r \in S_{c}$.

Proof. First we note that $N_{s} \in \operatorname{Irr} N(S)$ requires $N_{s}>1$, which amounts to $s \in S \backslash S_{c}$, see Proposition 2.4(i). Likewise, $s a=t r$ with $a \in S_{c}$ and $t \notin S_{c}$ or $r \notin S_{c}$ forces $s \notin S_{c}$. Thus we can restrict our attention to $s \in S \backslash S_{c}$. In case there are $a \in S_{c}$ and $t, r \in S \backslash S_{c}$ such that $s a=t r$, Proposition 2.4(i) entails that $N_{s}=N_{s a}=N_{t} N_{r}$ with $N_{t}, N_{r}>1$, which means $N_{s} \notin \operatorname{Irr} N(S)$. Conversely, if $N_{s} \notin \operatorname{Irr} N(S)$, then there are $t, r \in S$ with $t, r \in S \backslash S_{c}$ and $a \in S_{c}$ such that $s a=t r$, see Lemma 3.3.

Definition 4.2. Let $S$ be a right LCM monoid. An element $s \in S$ is noncore irreducible if $s \notin S_{c}$ and whenever $s a=t r$ for some $a \in S_{c}$ and $t, r \in S$, then $t \in S_{c}$ or $r \in S_{c}$. The collection of all noncore irreducible elements of $S$ is denoted by $\mathcal{I}(S)$.

Remark 4.3. The notion introduced in Definition 4.2 is not to be confused with core irreducibility from [ABLS17]: $s \in S$ is core irreducible if $s=t a$ with $t \in S, a \in S_{c}$ implies $a \in S^{*}$, the subgroup of invertible elements in $S$. We note that noncore irreducibility is preserved under core equivalence.

Definition 4.4. The core graph of a right LCM monoid $S$ is the undirected graph $\Gamma(S):=(V, E)$ with vertex set $V:=\mathcal{I}(S) / \sim$ and edge set $E:=\{([s],[t]) \mid s$ ก $t\}$.

We will simply write $\Gamma$ whenever this is unambiguous. For convenience, we recall that $\Gamma$ is characterized by its unique decomposition into coconnected components $\left(\Gamma_{i}\right)_{i \in I}$ with $\Gamma_{i}=\left(V_{i}, E_{i}\right)$. Such coconnected components are determined by the vertex set $V_{i}$ as $E_{i}=V_{i} \times V_{i} \cap E$. A natural characterization of coconnectedness is that the $V_{i}$ forms a maximal, connected subset of the complimentary graph $(V, V \times V \backslash E)$.

Notation 4.5. For $s \in \mathcal{I}(S)$, we denote by $i(s) \in I$ the index with $[s] \in V_{i(s)}$.

Recall from Lemma 2.7 that $a .[s]:=[a s]$ defines an action $\alpha: S_{c} \curvearrowright S / \sim$ by bijections.

Lemma 4.6. For $a \in S_{c}$ and $s \in S$, as $\in \mathcal{I}(S)$ holds if and only if $s \in \mathcal{I}(S)$, that is, the action $\alpha$ restricts to an action on $\mathcal{I}(S) /$.

Proof. Let $a \in S_{c}$ and $s \in S$. It is clear that $a s \in S_{c}$ if and only if $s \in S_{c}$, so we may restrict to the case where $s \in S \backslash S_{c}$. We will prove that $\alpha_{a}^{-1}([s]) \in \mathcal{I}(S) / \sim$ if and only if $[s] \in \mathcal{I}(S) / \sim$, which is equivalent to the original claim since $\mathcal{I}(S)$ is closed under core equivalence (essentially by definition) and $\alpha_{a}$ is bijective, see Lemma 2.7. Using Lemma 2.1, there are $b \in S_{c}$ and $s^{\prime} \in S \backslash S_{c}$ such that $s S \cap a S=s b S, s b=a s^{\prime}$. We claim that $s \in \mathcal{I}(S)$ if and only if $s^{\prime} \in \mathcal{I}(S)$, that is, $[s] \in \mathcal{I}(S) / \sim$ if and only if $\left[s^{\prime}\right]=\alpha_{a}^{-1}([s]) \in \mathcal{I}(S)$ : If there are $c \in S_{c}, t, r \in S \backslash S_{c}$ with $s^{\prime} c=t r$, then $s b c=$ $a s^{\prime} c=(a t) r$ implies $s \notin \mathcal{I}(S)$. Thus $s \in \mathcal{I}(S)$ implies $s^{\prime} \in \mathcal{I}(S)$. Conversely, if there 
are $c \in S_{c}, t, r \in S$ with $s c=t r$, then $b S_{c} \cap c S_{c}=b c^{\prime} S_{c}, b c^{\prime}=c b^{\prime}$ for some $b^{\prime}, c^{\prime} \in S_{c}$ by Lemma 2.1. With $t r b^{\prime}=s c b^{\prime}=a s^{\prime} c^{\prime}$ we get a diagram

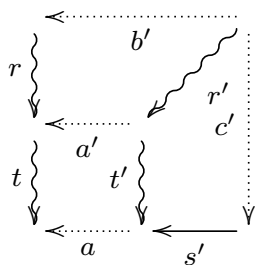

where $t S \cap a S=t a^{\prime} S, t a^{\prime}=a t^{\prime}$ and $r^{\prime} \in S$ with $r b^{\prime}=a^{\prime} r^{\prime}$. Here $r^{\prime}$ results from the fact that $t a^{\prime}$ is the right LCM of $t$ and $a$, while $t r b^{\prime}=a s^{\prime} c^{\prime}$ is another common right multiple. The dotted arrows represent elements from $S_{c}$, while solid arrows refer to $\mathcal{I}(S)$ (and $\rightsquigarrow$ bear no constraints). We get $s^{\prime} c^{\prime}=t^{\prime} r^{\prime}$ (upon using left cancellation). Since $a \in S_{c}$, we have $a^{\prime} \in S_{c}$ by Lemma 2.1. Thus $t \in S_{c}$ holds iff $t^{\prime} \in S_{c}$, and $r \in S_{c}$ iff $r^{\prime} \in S_{c}$. Therefore $s^{\prime} \in \mathcal{I}(S)$ forces $s \in \mathcal{I}(S)$.

Proposition 4.7. The action $\alpha: S_{c} \curvearrowright S / \sim$ determines an action $\beta: S_{c} \curvearrowright \Gamma$ by graph automorphisms. In particular, $\beta_{a}\left(\Gamma_{i}\right)$ is a coconnected component of $\Gamma$ isomorphic with $\Gamma_{i}$ for all $i \in I$ and $a \in S_{c}$.

Proof. By Lemma 4.6, $\alpha$ restricts to an action on the vertex set $\mathcal{I}(S) / \sim$. Recall that for $r, s, t \in S$ we have $s \perp t$ if and only if $r s \perp r t$ (using left cancellation). In particular, $([s],[t]) \in E$ is equivalent to $([a s],[a t]) \in E$ for all $s, t \in \mathcal{I}(S), a \in S_{c}$. Therefore $\alpha_{a}$ induces an automorphism $\beta_{a}$ of the graph $\Gamma$. For every such map, $\beta_{a}\left(\Gamma_{i}\right)$ is again a coconnected component of $\Gamma$ as these are determined by the connectivity inside the graph $\Gamma$.

As an immediate consequence of Proposition 4.7, we note:

Corollary 4.8. If the coconnected components of $\Gamma$ are mutually nonisomorphic, then $\alpha$ restricts to an action $\alpha_{a}: S_{c} \curvearrowright V_{i}$ on the vertex sets of the coconnected components $\Gamma_{i}$ for all $i \in I$.

Definition 4.9. Let $S$ be a right LCM monoid.

(i) The semigroup $S$ is noncore factorable if every element in $S \backslash S_{c}$ is core equivalent to a finite product of noncore irreducibles.

(ii) The semigroup $S$ has balanced factorization if $i(s) \neq i(t)$ implies $s S \cap t S=$ $s t^{\prime} S, s t^{\prime}=t s^{\prime}$ for some $s^{\prime}, t^{\prime} \in \mathcal{I}(S)$ with $i\left(s^{\prime}\right)=i(s), i\left(t^{\prime}\right)=i(t)$ for all $s, t \in \mathcal{I}(S)$, where $i(s) \in I$ is the index of the coconnected component of $\Gamma$ containing $[s]$.

Our next target is to detect the existence of and compute a right LCM for two finite products of noncore irreducibles.

Example 4.10. Assume that balanced factorization holds for $S$ and that the coconnected components of $\Gamma$ are edge-free. Let $s_{1}, s_{2}, t_{1}, t_{2}, t_{3} \in \mathcal{I}(S)$ and consider $s:=s_{1} s_{2}, t:=$ $t_{1} t_{2} t_{3}$. Suppose for convenience that we already know that $s$ ก $t$ holds (otherwise the process would terminate at one of the steps to come, and thus signalling $s \perp t$ ). Then we must have $s_{1} \cap t_{1}$ because $s S \subset s_{1} S$ and $t S \subset t_{1} S$. Suppose [ $\left.s_{1}\right]$ and $\left[t_{1}\right]$ belong to the same coconnected component of $\Gamma$. As the component is edge-free, this forces 
$s_{1} \sim t_{1}$, so there are $s_{1}^{(1)}, t_{1}^{(1)} \in S_{c}$ with $s_{1} S \cap t_{1} S=s_{1} t_{1}^{(1)} S, s_{1} t_{1}^{(1)}=t_{1} s_{1}^{(1)}$. Next, we will determine a right LCM for $s_{1}^{(1)}$ and $t_{2}$ : Since $s_{1}^{(1)} \in S_{c}$, Lemma 2.1 implies that there are $s_{1}^{(2)} \in S_{c}$ and $t_{2}^{(1)} \in S$ with $s_{1}^{(1)} S \cap t_{2} S=s_{1}^{(1)} t_{2}^{(1)} S, s_{1}^{(1)} t_{2}^{(1)}=t_{2} s_{1}^{(2)}$. Due to Lemma 4.6, we also know that $t_{2}^{(1)} \in \mathcal{I}(S)$. A repetition of this argument yields a right LCM for $s_{1}^{(2)}$ and $t_{3}: s_{1}^{(3)} \in S_{c}$ and $t_{3}^{(1)} \in \mathcal{I}(S)$ with $s_{1}^{(2)} S \cap t_{3} S=s_{1}^{(2)} t_{3}^{(1)} S, s_{1}^{(2)} t_{3}^{(1)}=t_{3} s_{1}^{(3)}$. This completes the first column in our diagram, where solid arrows refer to $\mathcal{I}(S)$ and dotted ones to $S_{c}$, as in the proof of Lemma 4.6:
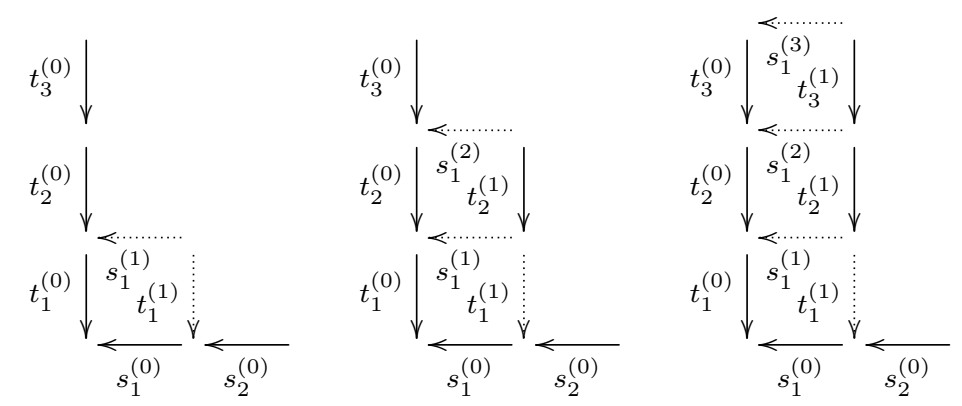

For the bottom square in the second column, the same argument yields a right LCM for $s_{2}$ and $t_{1}^{(1)}: s_{2}^{(1)} \in \mathcal{I}(S)$ and $t_{1}^{(2)} \in S_{c}$ with $s_{2} S \cap t_{1}^{(1)} S=s_{2} t_{1}^{(2)} S, s_{2} t_{1}^{(2)}=t_{1}^{(1)} s_{2}^{(1)}$. Thus we see that the encounter of a square with $s_{k+1}^{(\ell)} \sim t_{\ell+1}^{(k)}$ simplifies the task tremendously because we can then easily complete the corresponding row and column. Next, we focus on a right LCM for $s_{2}^{(1)}$ and $t_{2}^{(1)}$ : The assumption $s \cap t$ implies $s_{2}^{(1)} \cap t_{2}^{(1)}$. Suppose that $i\left(s_{2}^{(1)}\right) \neq i\left(t_{2}^{(1)}\right)$, that is, they belong to distinct coconnected components of $\Gamma$. Then balanced factorization grants $s_{2}^{(2)}, t_{2}^{(2)} \in \mathcal{I}(S)$ (with $i\left(s_{2}^{(1)}\right)=i\left(s_{2}^{(2)}\right)$ and $i\left(t_{2}^{(1)}\right)=i\left(t_{2}^{(2)}\right)$ ) such that $s_{2}^{(1)} S \cap t_{2}^{(1)} S=s_{2}^{(1)} t_{2}^{(2)} S, s_{2}^{(1)} t_{2}^{(2)}=t_{2}^{(1)} s_{2}^{(2)}$. For the right LCM for $s_{2}^{(2)}$ and $t_{3}^{(1)}$ suppose again that they have the same coconnected component, and thus $s_{2}^{(2)} \sim t_{3}^{(1)}$. This leads to the existence of $s_{2}^{(3)}, t_{3}^{(2)} \in S_{c}$ with $s_{2}^{(2)} S \cap t_{3}^{(1)} S=s_{2}^{(2)} t_{3}^{(2)} S, s_{2}^{(2)} t_{3}^{(2)}=t_{3}^{(1)} s_{2}^{(3)}$, and our diagram is complete:
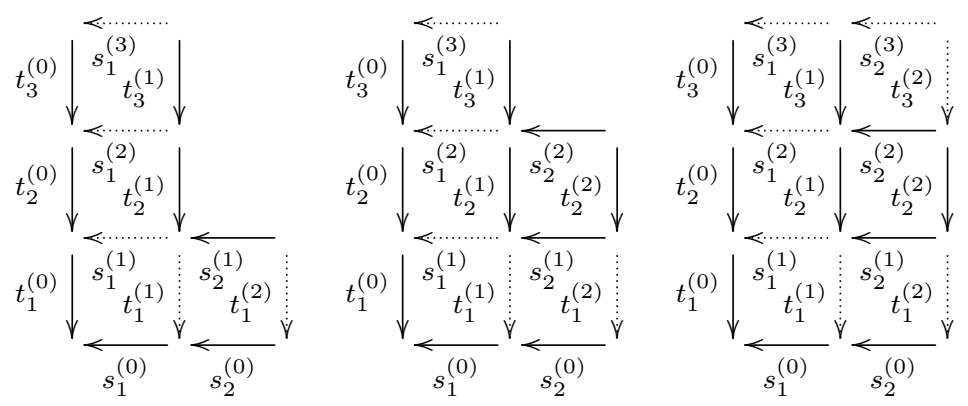

A right LCM for $s$ and $t$ is now given by $s t_{1}^{(2)} t_{2}^{(2)} t_{3}^{(2)}$ (or taking the product along any path from the upper right to the lower left corner in the completed diagram).

Remark 4.11. Let $s_{1}, \ldots, s_{m}, t_{1}, \ldots, t_{n} \in \mathcal{I}(S)$ and $s:=s_{1} \cdots s_{m}, t:=t_{1} \cdots t_{n}$. Assume that balanced factorization holds for $S$ and that the coconnected components of $\Gamma$ are edge-free. We want to describe an algorithm that decides whether $s \cap t$ or not, and produces a right LCM for $s$ and $t$ in case $s \cap t$. Let us first fix the notation: We introduce two indices $k, \ell$ with initial value $(k, \ell):=(0,0)$ and range $0 \leq k \leq m-1,0 \leq$ 
$\ell \leq n-1$, and set $s_{j}^{(0)}:=s_{j}$ for $1 \leq j \leq m, t_{j}^{(0)}:=t_{j}$ for $1 \leq j \leq n$. In order to find a right LCM for $s$ and $t$, we need to find elements $s_{k+1}^{(\ell)}, t_{\ell+1}^{(k)} \in \mathcal{I}(S) \cup S_{c}$, where $(0,0) \leq(k, \ell) \leq(m-1, n-1)$, such that

$$
s_{k+1}^{(\ell)} S \cap t_{\ell+1}^{(k)} S=s_{k+1}^{(\ell)} t_{\ell+1}^{(k+1)} S, \quad s_{k+1}^{(\ell)} t_{\ell+1}^{(k+1)}=t_{\ell+1}^{(k)} s_{k+1}^{(\ell+1)}
$$

holds for all $(0,0) \leq(k, \ell) \leq(m-1, n-1)$. In other words, we successively compute right LCMs for the (altered) factors of $s$ and $t$, and compose these to a right LCM of the product. We find it convenient to employ a flowchart for describing this repetitive process:

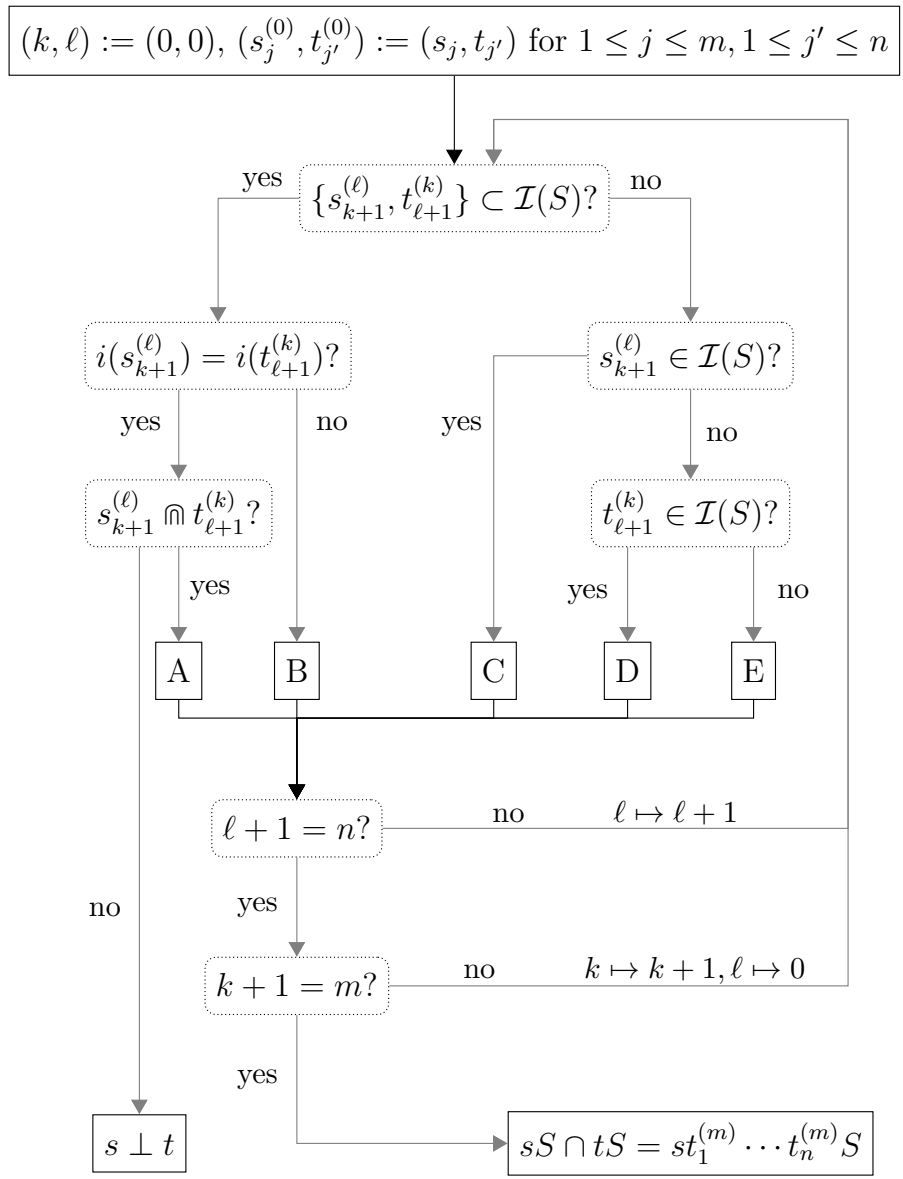

Before explaining the output, let us first describe the processes $A-E$ from the flowchart above, in which we compute a right LCM (or rather the missing right factors) for $s_{k+1}^{(\ell)}$ and $t_{\ell+1}^{(k)}$. Apart from $E$, these did already make an appearance in Example 4.10. We point out that $s_{k+1}^{(\ell)} \cap t_{\ell+1}^{(k)}$ holds whenever we enter any of these processes within the algorithm.

$A$ We have $s_{k+1}^{(\ell)}, t_{\ell+1}^{(k)} \in \mathcal{I}(S)$ such that $\left[s_{k+1}^{(\ell)}\right]$ and $\left[t_{\ell+1}^{(k)}\right]$ belong to the same coconnected component of $\Gamma$, that is, $i\left(s_{k+1}^{(\ell)}\right)=i\left(t_{\ell+1}^{(k)}\right)$. Then $s_{k+1}^{(\ell)} \cap t_{\ell+1}^{(k)}$ and the assumption of edge-freeness force $s_{k+1}^{(\ell)} \sim t_{\ell+1}^{(k)}$, so Lemma 2.2 implies:

$\rightsquigarrow$ There exist $s_{k+1}^{(\ell+1)}, t_{\ell+1}^{(k+1)} \in S_{c}$ with (4.1). 
$B$ We have $s_{k+1}^{(\ell)}, t_{\ell+1}^{(k)} \in \mathcal{I}(S)$ and the two vertices $\left[s_{k+1}^{(\ell)}\right],\left[t_{\ell+1}^{(k)}\right]$ belong to distinct coconnected components of $\Gamma$, that is, $i\left(s_{k+1}^{(\ell)}\right) \neq i\left(t_{\ell+1}^{(k)}\right)$. Here, balanced factorization implies:

$\rightsquigarrow$ There exist $s_{k+1}^{(\ell+1)}, t_{\ell+1}^{(k+1)} \in \mathcal{I}(S)$ with (4.1) satisfying $i\left(s_{k+1}^{(\ell)}\right)=i\left(s_{k+1}^{(\ell+1)}\right)$ and $i\left(t_{\ell+1}^{(k)}\right)=i\left(t_{\ell+1}^{(k+1)}\right)$.

$C$ We have $s_{k+1}^{(\ell)} \in \mathcal{I}(S)$ and $t_{\ell+1}^{(k)} \in S_{c}$. Thus Lemma 2.1 and Lemma 4.6 imply:

$\rightsquigarrow$ There are $s_{k+1}^{(\ell+1)} \in \mathcal{I}(S), t_{\ell+1}^{(k+1)} \in S_{c}$ with (4.1).

$D$ We have $s_{k+1}^{(\ell)} \in S_{c}$ and $t_{\ell+1}^{(k)} \in \mathcal{I}(S)$. Thus Lemma 2.1 and Lemma 4.6 imply:

$\rightsquigarrow$ There are $s_{k+1}^{(\ell+1)} \in S_{c}, t_{\ell+1}^{(k+1)} \in \mathcal{I}(S)$ with (4.1).

$E$ We have $s_{k+1}^{(\ell)}, t_{\ell+1}^{(k)} \in S_{c}$. So Lemma 2.1 implies:

$\rightsquigarrow$ There are $s_{k+1}^{(\ell+1)}, t_{\ell+1}^{(k+1)} \in S_{c}$ with $(4.1)$.

The algorithm starts with loop index $(k, \ell)=(0,0)$ and ends if it arrives at some level $\left(k^{\prime}, \ell^{\prime}\right)$ with $i\left(s_{k^{\prime}+1}^{\left(\ell^{\prime}\right)}\right)=i\left(t_{\ell^{\prime}+1}^{\left(k^{\prime}\right)}\right)$ and $s_{k^{\prime}+1}^{\left(\ell^{\prime}\right)} \perp t_{\ell^{\prime}+1}^{\left(k^{\prime}\right)}$, or if a right LCM has been obtained for $s_{m}^{(n-1)} \perp t_{n}^{(m-1)}$. In the first case, the algorithm shows

$$
s S \cap t S \subset s_{1} \cdots s_{k^{\prime}+1} S \cap t_{1} \cdots t_{\ell^{\prime}+1} S=s_{1} \cdots s_{k^{\prime}} t_{1}^{\left(k^{\prime}\right)} \cdots t_{\ell^{\prime}}^{\left(k^{\prime}\right)}\left(s_{k^{\prime}+1}^{\left(\ell^{\prime}\right)} S \cap t_{\ell^{\prime}+1}^{\left(k^{\prime}\right)} S\right)=\emptyset,
$$

which forces $s \perp t$. Similarly, the second case leads to

$$
s S \cap t S=s_{1} \cdots s_{m} t_{1}^{(m)} \cdots t_{n}^{(m)} S=t_{1} \cdots t_{n} s_{1}^{(n)} \cdots s_{m}^{(n)} S,
$$

in which case we obtain the right LCM for $s$ and $t$ by combining the right LCMs for the pairs appearing in the algorithm along a path on the grid from $(0,0)$ to $(m, n)$, compare Example 4.10.

Lemma 4.12. Suppose that all coconnected components of $\Gamma$ are edge-free and mutually nonisomorphic, and that $S$ has balanced factorization.

(i) Assume in addition that all coconnected components of $\Gamma$ are finite. If $s_{1}, \ldots, s_{n} \in$ $\mathcal{I}(S)$ and $\sigma$ is a permutation of $\{1, \ldots, n\}$, then there exist $t_{1}, \ldots, t_{n} \in \mathcal{I}(S)$ with $s_{1} s_{2} \cdots s_{n} \sim t_{1} \cdots t_{n}$ and $i\left(t_{k}\right)=i\left(s_{\sigma(k)}\right)$ for all $k$.

(ii) Let $m, n \geq 1$. Whenever $s_{1}, \ldots, s_{m}, t_{1}, \ldots, t_{n} \in \mathcal{I}(S)$ satisfy $s_{1} \cdots s_{m} \sim t_{1} \cdots t_{n}$, then $m=n$ and there is a permutation $\sigma$ of $\{1, \ldots, n\}$ such that $i\left(t_{\ell}\right)=i\left(s_{\sigma(\ell)}\right)$ for every $1 \leq \ell \leq n$.

Proof. For (i), let $s_{1}, \ldots, s_{n} \in \mathcal{I}(S), s:=s_{1} \cdots s_{n}$. Suppose that $\sigma_{1}, \sigma_{2}$ are permutations of $\{1, \ldots, n\}$ and $t_{1}^{(1)}, \ldots, t_{n}^{(1)}, t_{1}^{(2)}, \ldots, t_{n}^{(2)} \in \mathcal{I}(S)$ satisfy

(1) $s \sim t_{1}^{(1)} \cdots t_{n}^{(1)}$ with $i\left(t_{k}^{(1)}\right)=i\left(s_{\sigma_{1}(k)}\right)$ for all $k$; and

(2) $t_{1}^{(1)} \cdots t_{n}^{(1)} \sim t_{1}^{(2)} \cdots t_{n}^{(2)}$ with $i\left(t_{k}^{(2)}\right)=i\left(t_{\sigma_{2}(k)}^{(1)}\right)$ for all $k$.

Then we deduce that $t_{1}^{(2)}, \ldots, t_{n}^{(2)} \in \mathcal{I}(S)$ satisfies $s \sim t_{1}^{(2)} \cdots t_{n}^{(2)}$ with $i\left(t_{k}^{(2)}\right)=i\left(t_{\sigma_{2}(k)}^{(1)}\right)=$ $i\left(s_{\sigma_{1} \sigma_{2}(k)}\right)$ for all $k$. It therefore suffices to prove (i) for the Coxeter-Moore generators $\tau_{k}:=(k, k+1)$, where $1 \leq k \leq n-1$, because every permutation is a finite product of these. So let $1 \leq k \leq n-1$ and consider $\tau_{k}$ for $s:=s_{1} \cdots s_{n}$ with $s_{1}, \ldots, s_{n} \in \mathcal{I}(S)$. If $i\left(s_{k}\right)=i\left(s_{k+1}\right)$ holds, then $t_{\ell}:=s_{\ell}$ for all $\ell$ is a solution. In the case of $i\left(s_{k}\right) \neq i\left(s_{k+1}\right)$, we start by choosing $t_{j}:=s_{j}$ for $1 \leq j \leq k-1$. At step $k$, we need to invoke our assumptions: 
For every $t \in \mathcal{I}(S)$ with $i(t)=i\left(s_{k+1}\right) \neq i\left(s_{k}\right)$, there is an edge $\left([t],\left[s_{k}\right]\right)$ in $\Gamma$, that is, $t \cap s_{k}$. Thus balanced factorization yields elements $s_{t}, t^{\prime} \in \mathcal{I}(S)$ with $s_{k} S \cap t S=$ $s_{k} t^{\prime} S, s_{k} t^{\prime}=t s_{t}$ and $i\left(s_{t}\right)=i\left(s_{k}\right), i\left(t^{\prime}\right)=i(t)=i\left(s_{k+1}\right)$. For all $r, t \in \mathcal{I}(S)$ with $i(t)=i(r)=i\left(s_{k+1}\right)$ and $[t] \neq[r]$, edge-freeness of the coconnected components implies $t \perp r$. Therefore we get $s_{k} t^{\prime}=t s_{t} \perp r s_{r}=s_{k} r^{\prime}$, which is equivalent to $t^{\prime} \perp r^{\prime}$ by left cancellation. Hence $\left[t^{\prime}\right]$ and $\left[r^{\prime}\right]$ are distinct vertices from the coconnected component $\Gamma_{i\left(s_{k+1}\right)}$. Since the vertex set of $\Gamma_{i\left(s_{k+1}\right)}$ is finite, the map $t \mapsto t^{\prime}$ is a bijection, so that there is $t \in \mathcal{I}(S)$ with $i(t)=i\left(s_{k+1}\right)$ and $\left[t^{\prime}\right]=\left[s_{k+1}\right]$. Every such $t$ satisfies $s_{k} s_{k+1} \sim s_{k} t^{\prime}=t s_{t}$, say $s_{k} s_{k+1} S \cap t s_{t} S=s_{k} s_{k+1} a S, s_{k} s_{k+1} a=t s_{t} b$ for some $a, b \in S_{c}$. By setting $\left(t_{k}, t_{k+1}\right):=\left(t, s_{t} b\right)$ for such a $t$ which is fixed from now on (the class $[t]$ is uniquely determined), we have $s_{k} s_{k+1} a=t_{k} t_{k+1}$ for some $a \in S_{c}$ and $i\left(t_{k}\right)=i\left(s_{k+1}\right)$. Since $t_{k+1}=s_{t} b \sim s_{t}$ and $i\left(s_{t}\right)=i\left(s_{k}\right)$, we also get $i\left(t_{k+1}\right)=i\left(s_{k}\right)$.

Using left cancellation, $t_{j}=s_{j}$ for $1 \leq j \leq k-1$, and $t_{k} t_{k+1}=t s_{t} b=s_{k} s_{k+1} a$, we arrive at

$$
t_{1} \cdots t_{k+1} S \cap s_{1} \cdots s_{n} S=s_{1} \cdots s_{k+1}\left(a S \cap s_{k+2} \cdots s_{n} S\right) .
$$

Note that this also entails $t_{1} \cdots t_{k+1} \cap s_{1} \cdots s_{n}$ due to $a \in S_{c}$. Since the coconnected components are mutually nonisomorphic, it follows from Corollary 4.8 that $a S \cap s_{k+2} S=$ $a t_{k+2} S, a t_{k+2}=s_{k+2} a_{k+2}$ for some $a_{k+2} \in S_{c}$ and $t_{k+2} \in \mathcal{I}(S)$ with $i\left(t_{k+2}\right)=i\left(s_{k+2}\right)$. Repeating this procedure for $\left(a_{j}, s_{j}\right)$ with $k+3 \leq j \leq n$ allows us to arrive at a set of elements $t_{k+3}, \ldots, t_{n} \in \mathcal{I}(S)$ with $i\left(t_{j}\right)=i\left(s_{j}\right)$ for all $j \geq k+2$ and

$$
\begin{aligned}
t_{1} \cdots t_{n} & =s_{1} \cdots s_{k+1} a t_{k+2} t_{k+3} \cdots t_{n} \\
& =s_{1} \cdots s_{k+1} s_{k+2} a_{k+2} t_{k+3} \cdots t_{n} \\
& \vdots \\
& =s_{1} \cdots s_{n} a_{n} \\
& \sim s_{1} \cdots s_{n} .
\end{aligned}
$$

This completes the proof of (i) as $i\left(t_{j}\right)=i\left(s_{\tau_{k}(j)}\right)$ holds for all $1 \leq j \leq n$ with this choice of $t_{1}, \ldots, t_{n} \in \mathcal{I}(S)$.

For (ii), let $s:=s_{1} \cdots s_{m}$ and $t:=t_{1} \cdots t_{n}$ and suppose $s \sim t$. Then $s S \cap t S=$ $s a S, s a=t b$ for some $a, b \in S_{c}$, see Lemma 2.2. Thus Remark 4.11 states that $s S \cap$ $t S=s t_{1}^{(m)} \cdots t_{n}^{(m)} S=s a S$, which forces $t_{1}^{(m)} \cdots t_{n}^{(m)} \in S_{c}$. As $S_{c}$ is hereditary, see Lemma 2.2, we get $t_{\ell}^{(m)} \in S_{c}$ for every $1 \leq \ell \leq n$. Recall from Remark 4.11 that $t_{\ell}^{(0)}=t_{\ell} \in \mathcal{I}(S) \subset S \backslash S_{c}$, and that $t_{\ell}^{(k)} \in S_{c}$ implies $t_{\ell}^{\left(k^{\prime}\right)} \in S_{c}$ for all $k \leq k^{\prime} \leq m$ since the flowchart will always end up in process $C$ or $E$ for these cases. It follows that, for each $1 \leq \ell \leq n$, there is a unique minimal $k_{\ell} \geq 1$ such that $t_{\ell}^{\left(k_{\ell}\right)} \in S_{c}$, but $t_{\ell}^{(k)} \notin S_{c}$ for $0 \leq k<k_{\ell}$.

We will now argue that this entails a bijection $\{1, \ldots, m\} \rightarrow\{1, \ldots, n\}$ : According to Remark 4.11 , the only process that leads to an output $t_{\ell}^{\left(k_{\ell}\right)}$ in the core starting from $t_{\ell}^{\left(k_{\ell}-1\right)} \in \mathcal{I}(S)$ is $A$. Thus we must have $t_{\ell}^{\left(k_{\ell}-1\right)} \sim s_{k_{\ell}}^{(\ell-1)}$ for all $\ell$. In particular, this implies that

$$
s_{k_{\ell}}^{(\ell-1)} \in \mathcal{I}(S) \text { with } i\left(t_{\ell}^{\left(k_{\ell}-1\right)}\right)=i\left(s_{k_{\ell}}^{(\ell-1)}\right) .
$$

Moreover, it follows that the map $\sigma:\{1, \ldots, m\} \rightarrow\{1, \ldots, n\}, \ell \mapsto k_{\ell}$ is injective: For every $\ell^{\prime}>\ell$, we have $s_{k_{\ell}}^{\left(\ell^{\prime}-1\right)} \in S_{c}$ because of $s_{k_{\ell}}^{(\ell)} \in S_{c}$. Therefore, the right LCM of 
$s_{k_{\ell}}^{\left(\ell^{\prime}-1\right)}$ and $t_{\ell^{\prime}}^{\left(k_{\ell}-1\right)}$ will be obtained either through process $D$ or $E$. In the first case we get $t_{\ell^{\prime}}^{\left(k_{\ell}\right)} \notin S_{c}$, while the latter case requires $t_{\ell^{\prime}}^{\left(k_{\ell}-1\right)} \in S_{c}$, which forces $k_{\ell^{\prime}}<k_{\ell}$ due to minimality $k_{\ell^{\prime}}$.

Switching the role of the $s_{i}$ and the $t_{j}$, we get an injection $\sigma^{\prime}:\{1, \ldots, n\} \rightarrow\{1, \ldots, m\}$, $k \mapsto \ell_{k}$. Together with the injective map $\sigma$, this shows $m=n$. Moreover, $s_{k_{\ell}}^{(\ell-1)} \in \mathcal{I}(S)$ satisfies $i\left(t_{\ell}^{\left(k_{\ell}-1\right)}\right)=i\left(s_{k_{\ell}}^{(\ell-1)}\right)$, allowing us to deduce $\ell_{k_{\ell}}=\ell$, that is, $\sigma^{\prime} \circ \sigma=\mathrm{id}$.

Next, let us note that

(a) process $B$ preserves the coconnected components due to balanced factorization, that is, $i\left(s_{k+1}^{(\ell)}\right)=i\left(s_{k+1}^{(\ell+1)}\right)$ and $i\left(t_{\ell+1}^{(k)}\right)=i\left(t_{\ell+1}^{(k+1)}\right)$; and that

(b) processes $C$ and $D$ preserve the coconnected component of the respective noncore irreducible element due to mutually nonisomorphic coconnected components, see Corollary 4.8 .

Since only the processes $B, C$, or $D$ have occured for computing the right LCM of $s_{k}^{(\ell-1)}$ and $t_{\ell}^{(k-1)}$ with $k<k_{\ell}$, and then $A$ takes place for $k=k_{\ell}-1$, we deduce from (a) and (b) that

$$
i\left(t_{\ell}\right)=i\left(t_{\ell}^{\left(k_{\ell}-1\right)}\right)=i\left(s_{k_{\ell}}^{(\ell-1)}\right)=i\left(s_{k_{\ell}}\right)=i\left(s_{\sigma(\ell)}\right) \quad \text { holds for all } 1 \leq \ell \leq n .
$$

Theorem 4.13. A right LCM monoid $S$ admits a generalized scale if and only if the following conditions hold:

(i) The family $\left(\left|V_{i}\right|\right)_{i \in I} \subset \mathbb{N}^{\times} \cup\{\infty\}$ freely generates a nontrivial submonoid of $\mathbb{N}^{\times}$.

(ii) The coconnected components $\Gamma_{i}$ are edge-free.

(iii) $S$ is noncore factorable.

(iv) $S$ has balanced factorization.

If $S$ satisfies (i)-(iv), then the generalized scale $N: S \rightarrow \mathbb{N}^{\times}$is determined by $N_{s}:=\left|V_{i}\right|$ for $[s] \in V_{i}$.

Proof. Suppose first that there is a generalized scale $N$ on $S$. For $s, t \in \mathcal{I}(S)$ with $s \not t$, Proposition 2.4(ii) shows $([s],[t]) \notin E$ whenever $N_{s}=N_{t}$. Thus the coconnected component $\Gamma_{i}=\left(V_{i}, E_{i}\right)$ with $[s] \in V_{i}$ satisfies $N^{-1}\left(N_{s}\right) / \sim \subset V_{i}$. On the other hand, if $N_{s} \neq N_{t}$, then Lemma 3.4 shows $s$ ก $t$, that is, $([s],[t]) \in E$, because $N_{s}, N_{t} \in \operatorname{Irr} N(S)$ by Proposition 4.1. Thus we see that the coconnected components of $\Gamma(S)$ are given by $\left(\left(N^{-1}(n) / \sim, \emptyset\right)\right)_{n \in \operatorname{Irr} N(S)}$. This shows (ii) and also (i), as $\operatorname{Irr} N(S)$ freely generates $N(S)$ by Proposition 3.1.

Property (iii) is a direct consequence of Lemma 3.5. Concerning (iv), if $s, t \in \mathcal{I}(S)$ satisfy $N_{s} \neq N_{t}$, then $s \cap t$ by Proposition 4.1 and Lemma 3.4. Due to Proposition 2.4(iv) and Proposition 3.1, we have $s S \cap t S=s t^{\prime} S$, st $t^{\prime}=t s^{\prime}$ for some $s^{\prime}, t^{\prime} \in S$ satisfying $N_{s t^{\prime}}=N_{s} N_{t}$. This yields $N_{s^{\prime}}=N_{s}$ and $N_{t^{\prime}}=N_{t}$, and hence (iv).

Conversely, suppose that $S$ satisfies properties (i)-(iv). We first note that (i) forces $\left|V_{i}\right| \geq 2$ for all $i \in I$ and that these are all mutually distinct. Using (i) and (iii), we define $N: S \rightarrow \mathbb{N}^{\times}$by $\left.N\right|_{S_{c}}=1$ and $s \sim s_{1} \cdots s_{m} \mapsto \prod_{k=1}^{m}\left|V_{i\left(s_{k}\right)}\right|$, where $s_{k} \in \mathcal{I}(S)$ for all $k$. If $s \sim t_{1} \cdots t_{n}$ with $t_{\ell} \in \mathcal{I}(S)$ for all $\ell$, then Lemma 4.12(ii) implies that $m=n$ and that there is a permutation $\sigma$ of $\{1, \ldots, m\}$ such that $i\left(t_{\ell}\right)=i\left(s_{\sigma(\ell)}\right)$ for all $1 \leq \ell \leq m$. 
In particular, we get $\prod_{k=1}^{m}\left|V_{i\left(s_{k}\right)}\right|=\prod_{\ell=1}^{n}\left|V_{i\left(t_{\ell}\right)}\right|$, and hence $N$ is a well-defined map. It is then easy to see that it is in fact a homomorphism of monoids.

Suppose $s, t^{\prime} \in S$ satisfy $N_{s}=N_{t^{\prime}}$, and let $N_{s}=n_{1} \cdots n_{m}, n_{k}=\left|V_{i_{k}}\right|$, which is the unique factorization by (i). Due to (iii), $s \sim s_{1} \cdots s_{m}$ and $t^{\prime} \sim t_{1}^{\prime} \cdots t_{m}^{\prime}$ for some $s_{k}, t_{k}^{\prime} \in$ $\mathcal{I}(S)$. In addition, there exists a permutation $\sigma$ of $\{1, \ldots, m\}$ such that $i\left(s_{k}\right)=i\left(t_{\sigma(k)}^{\prime}\right)$ for all $k$. Invoking Lemma 4.12(i), we find $t_{1}, \ldots, t_{m} \in \mathcal{I}(S)$ with $i\left(t_{k}\right)=i\left(t_{\sigma(k)}^{\prime}\right)=$ $i\left(s_{k}\right)$ for all $k$ and $t^{\prime} \sim t_{1}^{\prime} \cdots t_{m}^{\prime} \sim t:=t_{1} \cdots t_{m}$. We can now apply the algorithm of Remark 4.11 to $s$ and $t$. For $m=4$ with $s \sim t$, the output diagram from Remark 4.11 would be

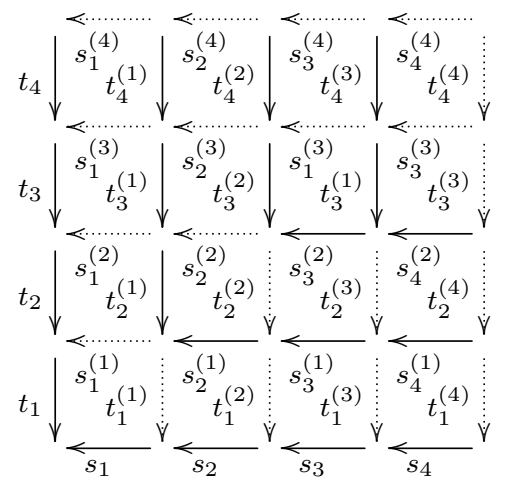

where dashed arrows refer to $S_{c}$ while solid arrows refer to $\mathcal{I}(S)$. More precisely, we get $s \perp t$, unless $s_{1} \sim t_{1}$ due to $i\left(s_{1}\right)=i\left(t_{1}\right)$ and (ii). This leads to $s_{1}^{(k)}, t_{1}^{(k)} \in S_{c}$ for all $k \geq 1$. By iteration, we see that we only need to check that

$$
s_{k}^{(k-1)} \sim t_{k}^{(k-1)}
$$

holds for all $1 \leq k \leq m$ to decide upon $s$ ก $t$, as all other squares will involve (precisely) one element from $S_{c}$ in its lower half. This stems from the fact that if (4.2) holds for $k=1, \ldots, k_{0}$, then we get $s_{k}^{(\ell)}, t_{k}^{(\ell)} \in S_{c}$ for all $1 \leq k \leq \ell \leq m, k \leq k_{0}$. Now suppose there is $\ell \leq m$ such that (4.2) holds for all $k<\ell$. According to the diagram obtained from Remark 4.11 up to $\ell-1$, (4.2) will hold for $\ell$ if and only if

$$
\alpha_{t_{\ell-1}^{(\ell-1)} \ldots t_{1}^{(\ell-1)}}^{-1}\left(\left[s_{\ell}\right]\right)=\left[s_{\ell}^{(\ell-1)}\right]=\left[t_{\ell}^{(\ell-1)}\right]=\alpha_{s_{\ell-1}^{(\ell-1)} \ldots s_{1}^{(\ell-1)}}^{-1}\left(\left[t_{\ell}\right]\right) .
$$

For convenience, let $a:=t_{\ell-1}^{(\ell-1)} \cdots t_{1}^{(\ell-1)}, b:=s_{\ell-1}^{(\ell-1)} \cdots s_{1}^{(\ell-1)}\left(\in S_{c}\right)$. By (i), the coconnected components have vertex sets of mutually distinct cardinalities, so they are mutually nonisomorphic. Thus Corollary 4.8 applies, showing that $\alpha_{a}$ and $\alpha_{b}$ restrict to bijections on $V_{i\left(s_{\ell}\right)}$ and $V_{i\left(t_{\ell}\right)}$, respectively. In particular, we have $i\left(s_{\ell}^{(\ell-1)}\right)=i\left(s_{\ell}\right)=$ $i\left(t_{\ell}\right)=i\left(t_{\ell}^{(\ell-1)}\right)$, so that $s_{\ell}^{(\ell-1)} \cap t_{\ell}^{(\ell-1)}$ is in fact equivalent to $s_{\ell}^{(\ell-1)} \sim t_{\ell}^{(\ell-1)}$.

If (4.2) holds for all $k$, that is, $s$ ก $t$, then the top row and the rightmost column of the diagram jointly mediate

$$
s \sim s_{1} \cdots s_{m} \sim s_{1} \cdots s_{m} t_{1}^{(m)} \cdots t_{m}^{(m)}=t s_{1}^{(m)} \cdots s_{m}^{(m)} \sim t \sim t^{\prime} .
$$

Thus $N$ satisfies (ii) of Definition 1.1, that is, $N_{s}=N_{t^{\prime}}$ implies $s \sim t^{\prime}$ or $s \perp t^{\prime}$. 
A second conclusion we draw from the previous consideration is that $\left|N^{-1}(n) / \sim\right|=n$ for all $n \in N(S)$ : For $n=n_{1} \cdots n_{m}, n_{k}=\left|V_{i_{k}}\right|$, we can pick

$$
\left\{s_{k, j} \mid 1 \leq k \leq m, 1 \leq j \leq n_{k}\right\} \subset \mathcal{I}(S)
$$

such that $i\left(s_{k, j}\right)=i_{k}$ and $s_{k, j} \perp s_{k, j^{\prime}}$ for all $j \neq j^{\prime}$. This already shows $\left|N^{-1}(n) / \sim\right| \geq n$ as $\left(s_{1, j_{1}} \cdots s_{m, j_{m}}\right)_{1 \leq j_{k} \leq n_{k}} \subset N^{-1}(n)$ consists of $n$ mutually orthogonal elements. Now for $[t] \in N^{-1}(n) / \sim$, we can assume that $t \sim t_{1} \cdots t_{m}$ with $i\left(t_{k}\right)=i_{k}$ for all $k$ (using the replacement technique for $t^{\prime} \mapsto t \sim t^{\prime}$ from before). We are going to find $\left(j_{1}, \ldots, j_{m}\right)$ such that $t \sim s_{1, j_{1}} \cdots s_{m, j_{m}}$ : As $n_{1}=\left|V_{i_{1}}\right|$, there is a unique $1 \leq j_{1} \leq n_{1}$ such that $t_{1} \sim s_{1, j_{1}}$. Therefore (4.2) holds for $k=1$ and we get $t_{1}^{(k)}, s_{1, j_{1}}^{(k)} \in S_{c}$ for all $1 \leq k \leq m$. As $\alpha_{t_{1}^{(k)}} \alpha_{s_{1, j_{1}}^{(1)}}^{-1}$ restricts to a bijection of $V_{i_{2}}$, see Corollary 4.8 , there is $1 \leq j_{2} \leq n_{2}$ such that $\alpha_{t_{1}^{(1)}} \alpha_{s_{1, j_{1}}^{(1)}}^{-1}\left(\left[t_{2}\right]\right)=\left[s_{2, j_{2}}\right]$, which is $(4.2)$ for $k=2$. Iterating this process, where at stage $k$ we find $1 \leq j_{k} \leq n_{k}$ with

$$
\alpha_{t_{k-1, j_{k-1}}^{(k-1)} \cdots t_{1, j_{1}}^{(1)}} \alpha_{s_{k-1, j_{k-1}}^{(k-1)} \cdots s_{1, j_{1}}^{(1)}}^{-1}\left(\left[t_{k}\right]\right)=\left[s_{k, j_{k}}\right]
$$

that gives (4.2). This shows $\left|N^{-1}(n) / \sim\right|=n$.

The above procedure is also useful in proving (iii) of Definition 1.1: Let $s \in S, n \in$ $N(S)$. Without loss of generality, we may assume $s \in S \backslash S_{c}, n>1$ as the remaining cases are trivial due to the presence of core elements. Then (iii) asserts that $s \sim s_{1} \cdots s_{m_{1}}$ for some $s_{k} \in \mathcal{I}(S)$ with $\left|V_{i\left(s_{k}\right)}\right|=: n_{1, k}$, while (i) gives $n=n_{2,1} \cdots n_{2, m_{2}}$ for uniquely determined $n_{2, k} \in\left\{\left|V_{i}\right| \mid i \in I\right\}$. By rearrangement of the factors $n_{2, k}$ and possibly switching $s \mapsto s^{\prime} \sim s$ via Lemma 4.12(i), we can assume that there is $0 \leq \ell \leq m_{1} \wedge m_{2}$ such that $n_{1, k}=n_{2, k}$ for $1 \leq k \leq \ell$, while $n_{1, k} \neq n_{2, k^{\prime}}$ for all $\ell<k, k^{\prime}$. In plain words, we extract the greatest common divisor of $n_{1,1} \cdots n_{1, m_{1}}$ and $n$. We then simply set $t_{k}:=s_{k}$ for $1 \leq k \leq \ell$ and fix an arbitrary choice of $t_{\ell+1}, \ldots, t_{m_{2}}$ with $n_{2, k}=\left|V_{i\left(t_{k}\right)}\right|$ for $\ell+1 \leq k \leq m_{2}$. Looking at Remark 4.11 for $s_{\ell+1} \cdots s_{m_{1}}$ and $t_{\ell+1}, \ldots, t_{m_{2}}$, we see that due to $n_{1, k} \neq n_{2, k^{\prime}}$ for all $\ell<k, k^{\prime}$, we can always complete the diagram as we only apply process $B$ in every step. The cause of this outcome is nothing but balanced factorization. Thus we find $t \in N^{-1}(n)$ with $s \cap t$ as required, and we have shown that $N$ is indeed a generalized scale.

Remark 4.14. Due to the explicit description of the generalized scale in terms of $\Gamma(S)$, Theorem 4.13 not only characterizes the existence of the generalized scale, but also implies uniqueness, which we already showed abstractly in Theorem 3.6.

\section{Examples, Challenges, and Saito's Degree map}

5.1. Self-similar group actions. Let $(G, X)$ be a self-similar group action, that is, $X$ is a finite alphabet in at least two letters and $G$ is a group acting on the free monoid $X^{*}$ generated by $X$ such that for each $x \in X, g \in G$, there are unique elements $g(x) \in$ $X,\left.g\right|_{x} \in G$ such that $g(x w)=\left.g(x) g\right|_{x}(w)$ for all $w \in X^{*}$. The associated Zappa-Szép product $S:=X^{*} \bowtie G$ is the monoid with elements $X^{*} \times G$ and product $(v, g)(w, h):=$ $\left(v g(w),\left.g\right|_{w} h\right)$, where the expression $\left.g\right|_{w}$ is defined recursively through $\left.g\right|_{x u}:=\left.\left(\left.g\right|_{x}\right)\right|_{u}$. It was observed that $S$ is a right LCM monoid in [BRRW14, Theorem 3.8], and that $S$ 
admits a generalized scale, see [ABLS17, Subsection 5.3]. We can recover the second part easily from Theorem 4.13:

(a) $S_{c}=S^{*}=\{(\varnothing, g) \mid g \in G\} \cong G$.

(b) An element $(w, g)$ belongs to $\mathcal{I}(S)$ if and only if $w \in X$.

(c) For every $(w, g) \in S \backslash S_{c}, w$ is a nontrivial word $w=x_{1} x_{2} \cdots x_{n}$ in $X$ of length $n \geq 1$. Thus $(w, g)=\left(x_{1}, 1\right)\left(x_{2}, 1\right) \cdots\left(x_{n-1}, 1\right)\left(x_{n}, g\right)$ shows that $S$ is noncore factorable.

(d) For $(x, g),(y, h) \in \mathcal{I}(S)$, we get $(x, g) \cap(y, h)$ if and only if $x=y$ if and only if $(x, g) \sim(y, h)$. Thus, $\Gamma(S)$ is the empty graph on $|X|$ vertices $[(x, 1)], x \in X$, which is coconnected.

Hence conditions (i)-(iv) in Theorem 4.13 are satisfied and $(w, g) \mapsto|X|^{\ell(w)}$ defines a generalized scale, where $\ell: X^{*} \rightarrow \mathbb{N}$ denotes the word length with respect to $X$.

5.2. The $\mathbf{a x}+\mathbf{b}$-semigroup over the natural numbers. A famous example of a right LCM monoid with a generalized scale is the ax+b-semigroup over the natural numbers $S:=\mathbb{N} \rtimes \mathbb{N}^{\times}$, see [ABLS17, Subsection 5.4]. This example features:

(a) $S_{c}=\{(n, 1) \mid n \in \mathbb{N}\} \cong \mathbb{N}$.

(b) An element $(m, p)$ belongs to $\mathcal{I}(S)$ if and only if $p$ is a prime.

(c) As every positive integer is a finite product of primes, $S$ is noncore factorable.

(d) Let $(m, p),(n, q) \in \mathcal{I}(S)$. If $p=q$, then $(m, p) \cap(n, q)$ holds if and only if $m-n \in p \mathbb{Z}$, that is, $(m, p) \sim(n, q)$. If $p \neq q$, then $p \mathbb{Z}+q \mathbb{Z}=\operatorname{gcd}(p, q) \mathbb{Z}=\mathbb{Z}$ and $(m, p) S \cap(n, q) S=\left(m+p n^{\prime}, p q\right) S, m+p n^{\prime}=n+q m^{\prime}$, where $m^{\prime}, n^{\prime} \in \mathbb{N}$ represent the smallest nonnegative solution for $m+p n^{\prime}=n+q m^{\prime}$ (the solution in $\mathbb{Z}$ is unique up to $p q \mathbb{Z})$. We thus get the coconnected components of $\Gamma(S)$ to be $\left(V_{p}\right)_{p \in P}$, where $P$ denotes the primes in $\mathbb{N}^{\times}$, with $V_{p}=\{[(k, p)] \mid 0 \leq k<p\}$ and $E_{p}=$.

(e) In this example, balanced factorization mirrors the fact that the prime factorization mentioned in (c) is unique (up to permutation of factors).

So Theorem 4.13 implies that $(m, p) \mapsto p$ is the unique generalized scale on $S$. In passing, we note that Theorem 4.13 and the notion of a generalized scale thereby provide a profound justification of "the obvious choice" made in [LR10] as described in [BLRS19, Remark 2.4].

5.3. Algebraic dynamical systems. Let $(G, P, \theta)$ be an algebraic dynamical system as considered in [BLS18], that is, $P$ is a right LCM monoid acting upon a discrete group $G$ by injective group endomorphisms $\theta_{p}$ such that $p P \cap q P=r P$ implies $\theta_{p}(G) \cap \theta_{q}(G)=$ $\theta_{r}(G)$. The right LCM monoid of interest here is $S:=G \rtimes_{\theta} P$, and we assume that $p \in P^{*}$ whenever $\theta_{p} \in \operatorname{Aut}(G)^{\mathrm{a}}$.

Remark 5.1. The set $\mathcal{I}\left(G \rtimes_{\theta} P\right)$ is given by $G \times \mathcal{I}(P)$. It is shown in [ABLS17, Proposition $5.11(\mathrm{i})]$ that $S$ admits a generalized scale (given by $N_{(g, p)}:=\left[G: \theta_{p}(G)\right]$ ) provided that:

(a) The index $N_{p}:=\left[G: \theta_{p}(G)\right]$ is finite for all $p \in P$, and $N_{p}>1$ for some $p \in P$.

(b) If $N_{p}=N_{q}$ for some $p, q \in P$, then $p \in q P^{*}$.

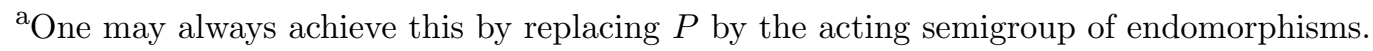


It is easy to see from Theorem 4.13(i) and (iii) that part (a) is also necessary: Suppose first that there was $p \in P$ with infinite index. For the semigroup $S=G \rtimes_{\theta} P$ to be noncore factorable, $P$ has to be noncore factorable, so $p$ would have to be expressible as a finite product $p=p_{1} q_{2} \cdots p_{n}$ for some $p_{k} \in \mathcal{I}(P)$. Since the index is multiplicative, there would exist $q \in \mathcal{I}(P)$ with infinite index. We observe that $(g, q) \in \mathcal{I}(S)$ for all $g \in G$ with $(g, q) \sim(h, q)$ if and only if $g^{-1} h \in \theta_{q}(G)$. Thus the coconnected component $\Gamma_{i}$ of $[(g, q)]$ will contain infinitely many vertices, thereby violating condition (i) of Theorem 4.13. So $P$ must act by finite index endomorphisms. If all of them are automorphisms, then $S$ is a group and hence $\Gamma=(\{v\}, \emptyset)$ will be an obstruction to nontriviality in Theorem 4.13 (i). The next example shows that part (b) is too strong in general:

Example 5.2 (Freely doubled $\times 2$ ). Let $P$ be the free monoid generated by two elements $p, q, G=\mathbb{Z}$, and $\theta_{p}=\theta_{q}=\times 2: \mathbb{Z} \rightarrow \mathbb{Z}$. Then $S=G \rtimes_{\theta} P$ is a right LCM monoid with $\mathcal{I}(S)=\{(n, r) \mid n \in \mathbb{Z}, r \in\{p, q\}\}$, see Remark 5.1. We note that $(m, p) \perp$ $(n, q)$ for all $m, n \in \mathbb{Z}$, while $(m, r) \cap(n, r)$ if and only if $m-n \in \theta_{r}(G)=2 \mathbb{Z}$, that is, $(m, r) \sim(n, r)$, for $r \in\{p, q\}$. Hence $\Gamma(S)$ is the empty graph on four vertices $[(0, p)],[(1, p)],[(0, q)],[(1, q)]$. Therefore, $\Gamma(S)$ is coconnected and conditions (i),(ii), and (iv) of Theorem 4.13 follow immediately, while (iii) boils down to Remark 5.1, noting that $p$ and $q$ generate $P$ by construction. Thus $S$ admits a generalized scale $N$ with $N_{(g, w)}=4^{\ell(w)}$, where $\ell$ denotes the word length on $P$ with respect to $\{p, q\}$.

On the other hand, $G \rtimes_{\theta} P$ may fail to have a generalized scale for $P \cong \mathbb{N}^{2}$ as soon as condition (b) from Remark 5.1 is violated:

Example 5.3 (Ledrappier's shift). Consider the right LCM monoid $S:=G \rtimes_{\sigma, \mathrm{id}+\sigma} \mathbb{N}^{2}$, where $G:=\bigoplus_{\mathbb{N}} \mathbb{Z} / 2 \mathbb{Z}$ and $\sigma$ is the right shift $\left(g_{0}, g_{1} \ldots\right) \mapsto\left(0, g_{0}, g_{1}, \ldots\right)$. In other words, $S$ is the semidirect product of the standard restricted wreath product $\mathbb{Z} / 2 \mathbb{Z} \imath \mathbb{N}$ by $\mathbb{N}$, where the latter action is given by id $+\sigma$. This monoid has close ties to the well-known Ledrappier shift from [Led78], see [Sta, Example 2.9] and [ER07, Section 11]. It is also an example of a right LCM monoid arising from an algebraic dynamical system in the sense of [BLS18]. The two generators for the $\mathbb{N}^{2}$-action $\sigma$ and id $+\sigma$ are independent ${ }^{\mathrm{b}}$ injective group endomorphisms whose images have index two. More precisely, we have $G=\sigma(G) \sqcup e_{0}+\sigma(G)=(\mathrm{id}+\sigma)(G) \sqcup e_{0}+(\mathrm{id}+\sigma)(G)$, where $e_{k}:=\left(\delta_{k, n}\right)_{n \in \mathbb{N}}$. This leads us to the following features:

(a) $S_{c}=S^{*}=G \times\{0\}$.

(b) An element $(g, m)$ belongs to $\mathcal{I}(S)$ if and only if $m \in\{\sigma$, id $+\sigma\}$.

(c) The semigroup $S$ is noncore factorable because $\langle\sigma$, id $+\sigma\rangle \cong \mathbb{N}^{2}$, see Remark 5.1.

(d) Let $(g, m),(h, n) \in \mathcal{I}(S)$. If $m=n$, then $(g, m) \cap(h, n)$ if and only if $g_{0}=$ $h_{0}$ if and only if $(g, m) \sim(h, n)$. For $m \neq n$, we always get $(g, m) \cap(h, n)$ with $(g, m) S \cap(h, n) S=\left(g m\left(h^{\prime}\right), m n\right) S, g m\left(h^{\prime}\right)=h n\left(g^{\prime}\right)$ for suitable $g^{\prime}, h^{\prime} \in G$ due to strong independence of $\sigma$ and id $+\sigma$, that is, $\sigma(G)+(\operatorname{id}+\sigma)(G)=G$, see [Sta15, Definition 1.3]. This means, $\Gamma(S)$ has two coconnected components

\footnotetext{
${ }^{b}$ This strong form of commutativity for injective group endomorphisms of discrete groups surfaced in [CV13], and was analysed more closely in [Sta15, Section 1] and [Sta, Section 2], exhibiting a close connection to $*$-commutativity from [AR97].
} 
$\Gamma_{i}, i=1,2$ given by $V_{1}:=\left\{[(g, \sigma)] \mid g \in\left\{0, e_{0}\right\}\right\}$ and $V_{2}:=\{[(g, \mathrm{id}+\sigma)] \mid g \in$ $\left.\left\{0, e_{0}\right\}\right\}$ with $E_{i}=\emptyset$.

(e) Balanced factorization holds because of independence, see (d).

We note that unlike for the previous examples from 5.1,5.2, and Example 5.4, Corollary 4.8 does not apply, but its conclusion holds nonetheless. The natural alternative to a generalized scale for $S$ is the homomorphism given by $g \mapsto 1$ for $g \in G$ and $\sigma \mapsto 2$, id $+\sigma \mapsto 2$. We expect the corresponding dynamics on $C^{*}(S)$ to have a KMSstate structure of the same kind as we have established for right LCM monoids admitting a generalized scale, see [ABLS17, Theorem 4.3] and [BLRSt].

While all the previous examples satisfy the conclusion of Corollary 4.8 that $\alpha: S_{c} \curvearrowright$ $S / \sim$ restricts to permutations on the vertex sets of the coconnected components of $\Gamma(S)$, the following is an easy example, where this is no longer the case. As this requires the existence of two distinct isomorphic coconnected components in $\Gamma(S)$, any such example will fail condition (i) of Theorem 4.13. It will thus not have a generalized scale, which indicates that despite the usefulness of the notion of generalized scales, we ought to consider more flexible analogues eventually.

Example 5.4 (Multiplication with flip). Let $p \in \mathbb{Z},|p| \geq 2$ and

$$
p_{0}:=\left(\begin{array}{ll}
p & 0 \\
0 & 1
\end{array}\right), p_{1}:=\left(\begin{array}{ll}
1 & 0 \\
0 & p
\end{array}\right), x:=\left(\begin{array}{ll}
0 & 1 \\
1 & 0
\end{array}\right) \in M_{2}(\mathbb{Z}) .
$$

Consider $S:=\mathbb{Z}^{2} \rtimes P$, where $P \subset M_{2}(\mathbb{Z})$ is the monoid generated by $p_{0}$ and $x$. Then $S$ is a right $\mathrm{LCM}$ monoid and $P=\left\langle p_{0}, p_{1}\right\rangle \rtimes\langle x\rangle \cong \mathbb{N}^{2} \rtimes \mathbb{Z} / 2 \mathbb{Z}$ with $x p_{0} x=p_{1}$. We obtain:

(a) $S_{c}=S^{*}=\mathbb{Z}^{2} \rtimes \mathbb{Z} / 2 \mathbb{Z}$.

(b) An element $(m, q)$ belongs to $\mathcal{I}(S)$ if and only if $q=p_{i} x^{\ell}$ for $i, \ell \in\{0,1\}$ as every element in $P$ admits a unique normal form $q=p_{k_{1}} p_{k_{2}} \cdots p_{k_{n}} x^{\ell}$ with $k_{j}, \ell \in\{0,1\}$.

(c) The normal form described in (b) implies that $S$ is noncore factorable.

(d) Let $\left(m, q_{0}\right),\left(n, q_{1}\right) \in \mathcal{I}(S)$. If $q_{i}=p_{k} x^{\ell_{i}}$ for some $k, \ell_{i} \in\{0,1\}$, then $\left(m, q_{0}\right)$ ก $\left(n, q_{1}\right)$ holds if and only if $m-n \in p_{k} \mathbb{Z}^{2}$, that is, $\left(m, q_{0}\right) \sim\left(n, q_{1}\right)$. On the other hand, if $q_{i}=p_{i} x^{\ell_{i}}$ for some $\ell_{i} \in\{0,1\}, i=1,2$, then $\left(m, q_{0}\right) \cap\left(n, q_{1}\right)$ always holds as $p_{1} \mathbb{Z}^{2}+p_{2} \mathbb{Z}^{2}=\mathbb{Z}^{2}$. Thus $\Gamma(S)$ is given by two coconnected components $\Gamma_{0}$ and $\Gamma_{1}$ with $V_{i}:=\left\{\left[\left(m, p_{0}\right)\right] \mid 0 \leq m<p e_{i}\right\}$ for $i=0,1$, where $e_{0}=(1,0), e_{1}=(0,1)$, and $E_{i}=\emptyset$.

(e) Balanced factorization is due to $p_{0} \mathbb{Z}^{2} \cap p_{1} \mathbb{Z}^{2}=p_{0} p_{1} \mathbb{Z}^{2}, p_{0} p_{1}=p_{1} p_{0}$.

For $\left[\left(\left(m_{0}, m_{1}\right), p_{0}\right)\right] \in V_{0}$, we get $\alpha_{x}\left(\left[\left(\left(m_{0}, m_{1}\right), p_{0}\right)\right]\right)=\left[\left(\left(m_{1}, m_{0}\right), p_{1}\right)\right] \in V_{1}$, so the flip switches the two coconnected components $\Gamma_{0}$ and $\Gamma_{1}$.

5.4. Graph products. Suppose $\Lambda=(W, F)$ is an undirected graph, and $\left(S_{w}\right)_{w \in W}$ is a family of right LCM monoids. The graph product $S\left(\Lambda,\left(S_{w}\right)_{w \in W}\right)$ is then defined as the quotient of the free monoid $*_{w \in W} S_{w}$ by the congruence generated by the relations $s t=t s$ for $s \in S_{v}, t \in S_{w}$ with $(v, w) \in F$. Whenever there is no ambiguity concerning the vertex semigroups $\left(S_{w}\right)_{w \in W}$, we shall simply write $S(\Lambda)$. This construction goes back to the case of groups first considered in [Gre90], and was studied for monoids in [VdC01, FK09]. Prominent examples of this kind are right-angled Artin monoids, which have already been of interest to operator algebraists, see for instance [CL02, CL07, ELR16, Sta18]. We would like to mention that extensive research has been conducted on such monoids 
under the name of trace monoids in connection theoretical computer science, see [DR95]. A standard means of studying the structure of graph products is the decomposition of the graph $\Lambda$ into its coconnected components $\Lambda_{i}, i \in I$. For convenience, let us recall that graph $(V, E)$ is called coconnected if the graph $(V, V \times V \backslash E)$ is connected, see also [Sta18, Subsection 2.2]. Let $\left(\Lambda_{j}\right)_{j \in J}$ be the decomposition into coconnected components for $\Lambda$, so that the graph product satisfies $S(\Lambda)=\bigoplus_{j \in J} S\left(\Lambda_{j}\right)$. Now for each $j \in J$, we let $\left(\Gamma_{i}\right)_{i \in I_{j}}$ with $\Gamma_{i}=\left(V_{i}, E_{i}\right)$ denote the decomposition of $\Gamma\left(S\left(\Lambda_{j}\right)\right)$ into its coconnected components.

Theorem 5.5. Let $\Lambda=(W, F)$ be an undirected graph and $\left(S_{w}\right)_{w \in W}$ a family of right LCM monoids. Then:

(i) $\mathcal{I}(S(\Lambda))=\bigsqcup_{j \in J} \mathcal{I}\left(S\left(\Lambda_{j}\right)\right) \oplus \bigoplus_{j^{\prime} \in J \backslash\{j\}} S\left(\Lambda_{j^{\prime}}\right)_{c}$.

(ii) The coconnected components of $\Gamma(S(\Lambda))$ are edge-free if and only if the coconnected components of $\Gamma\left(S\left(\Lambda_{j}\right)\right)$ are edge-free for all $j \in J$.

(iii) $S(\Lambda)$ is noncore factorable if and only if $S\left(\Lambda_{j}\right)$ is noncore factorable for all $j \in J$.

(iv) $S(\Lambda)$ has balanced factorization if and only if $S\left(\Lambda_{j}\right)$ has balanced factorization and $\alpha: S\left(\Lambda_{j}\right)_{c} \curvearrowright S\left(\Lambda_{j}\right) / \sim$ restricts to an action on the vertex sets of the individual coconnected components for all $j \in J$.

In particular, the graph product $S(\Lambda)$ has a generalized scale if and only if the properties (ii)-(iv) from Theorem 4.13 hold for every $S\left(\Lambda_{j}\right), j \in J$, and $\left(\left|V_{i}\right|\right)_{i \in \bigsqcup_{j \in J} I_{j}} \subset \mathbb{N}^{\times} \cup\{\infty\}$ freely generates a nontrivial submonoid of $\mathbb{N}^{\times}$.

Proof. Part (i) is a straightforward observation using the definition of $S(\Lambda)$, and (iii) then follows easily. For $s=\sum_{j \in J} s_{j} \in \mathcal{I}(S(\Lambda)$, we let $j(s) \in J$ be the unique index with $s_{j(s)} \in \mathcal{I}\left(S\left(\Lambda_{j(s)}\right)\right)$. Then $s, t \in \mathcal{I}(S(\Lambda)$ satisfy $s$ n $t$ if and only if $j(s) \neq j(t)$ or $j(s)=j(t)$ and $s_{j(s)} \cap t_{j(s)}$. Thus the coconnected components of $\Gamma(S(\Lambda))$ are given by $\left(\Gamma_{i}\right)_{i \in \bigsqcup_{j \in J} I_{j}}$. With this insight, (ii) is an immediate consequence. By (i), balanced factorization for every $j \in J$ is necessary, corresponding to the case of $s, t \in \mathcal{I}(S(\Lambda))$ with $j(s)=j(t)$. For $j(s) \neq j(t)$, the description in (i) yields $s S(\Lambda) \cap t S(\Lambda)=s t^{\prime} S(\Lambda)$, st $=t s^{\prime}$ with $t_{j}^{\prime} \in S\left(\Lambda_{j}\right)_{c}$ if and only if $j \neq j(t)$ and $s_{j}^{\prime} \in S\left(\Lambda_{j}\right)_{c}$ if and only if $j \neq j(s)$. At $j(t)$ we have $\left[t_{j(t)}^{\prime}\right]=\alpha_{s_{j(t)}}^{-1}\left(\left[t_{j(t)}\right]\right) \in \mathcal{I}\left(S\left(\Lambda_{j(t)}\right)\right)$, and similarly for $s^{\prime}$ at $j(s)$. Thus the necessary and sufficient condition for balanced factorization is obtained by adding the assumption that $\alpha: S\left(\Lambda_{j}\right)_{c} \curvearrowright S\left(\Lambda_{j}\right) / \sim$ restricts to an action on the vertex set $V_{i}$ of each coconnected component $\Gamma_{i}, i \in I_{j}$, for all $j \in J$, compare Corollary 4.8. This proves (iv), and the claim about the existence of generalized scales is now a consequence of Theorem 4.13.

Remark 5.6. The condition that $\left(\left|V_{i}\right|\right)_{i \in \bigsqcup_{j \in J} I_{j}} \subset \mathbb{N}^{\times} \cup\{\infty\}$ freely generates a nontrivial submonoid of $\mathbb{N}^{\times}$can also be expressed by saying that

(a) $\left(\left|V_{i}\right|\right)_{i \in I_{j}} \subset \mathbb{N}^{\times} \cup\{\infty\}$ freely generates a submonoid $M_{j}$ of $\mathbb{N}^{\times}$for all $j \in J$;

(b) $M_{j} \cap M_{j^{\prime}}=\{1\}$ for all $j, j^{\prime} \in J, j \neq j^{\prime}$; and

(c) there is $j \in J$ such that $M_{j}$ is nontrivial.

In particular, we see that a necessary condition for $S(\Lambda)$ to admit a generalized scale $N$ is that $S\left(\Lambda_{j}\right)$ admits a generalized scale for all $j \in J$ with nontrivial $M_{j}$. In this case, $N$ is determined by this family of generalized scales. 
According to Theorem 5.5, the study of generalized scales (or analogues thereof) on graph products can be reduced to the coconnected case. But the situation seems to be quite intricate, as the following example shows:

Example 5.7 (Graph products behaving badly). Let us consider the graph product for $\Lambda=(\{v, w\}, \emptyset)$ with

$$
S_{v}=\langle b\rangle \rtimes\langle q\rangle \cong \mathbb{N} \rtimes \mathbb{N} \cong\langle b\rangle \rtimes\langle q\rangle=S_{w}
$$

for $p, q \in \mathbb{N}_{\geq 2}$, that is, the free product of two one-dimensional subdynamics from 5.2. As $\Lambda$ is edge-free, $S:=S(\Lambda)$ has

(a) $S_{c}=S^{*}=\left\{1_{S}\right\}$; and

(b) $\mathcal{I}(S)=\{(a, 1),(0, p),(b, 1),(0, q)\}$ with $\Gamma(S)$ having two edges $([(a, 1)],[(0, p)])$ and $([(b, 1)],[(0, q)])$.

So $\Gamma(S)$ is coconnected but has edges, so $S$ does not satisfy (ii) of Theorem 4.13. Therefore $S$ does not admit a generalized scale even though both $S_{v}$ and $S_{w}$ do.

It appears that a natural choice for the substitute of the generalized scale in Example 5.7 would be obtained by combining the generalized scales on $S_{v}$ and $S_{w}$ suitably with regard to $\Lambda$. More precisely, we could consider the homomorphism determined by $(a, 1),(b, 1) \mapsto 2,(0, p) \mapsto 2 p$, and $(0, q) \mapsto 2 q$. In this specific example we might, for each coconnected component,

(a) count the number of connected components, and

(b) pick the generalized scale on each of the connected components instead.

Such problems cannot occur if we restrict our attention to the special case where all the vertex semigroups are isomorphic to $\mathbb{N}$, that is, right-angled Artin monoids $S(\Lambda)=: A_{\Lambda}^{+}$. Here, the essential obstruction to the existence of the generalized scale in the coconnected case is the presence of edges, as $\Gamma\left(A_{\Lambda}^{+}\right) \cong \Lambda$, see Theorem 4.13. This already follows from [Sta18, Corollary 4.9], though the approach taken there does not provide for a workaround for graphs with edges. In this respect, Theorem 4.13 is a clear improvement: If $\Lambda$ is a coconnected graph with at least one edge (hence at least two vertices), then $A_{\Lambda}^{+}$satisfies all but condition (ii) of Theorem 4.13.

5.5. Saito's degree map. If $S$ admits a generalized scale $N: S \rightarrow \mathbb{N}^{\times}$, applying the logarithm results in a homomorphism deg: $S \rightarrow \mathbb{R}_{\geq 0}$. Under the assumption that the core $S_{c}$ is nothing more than the invertible elements $S^{*}$ of $S$, the map deg happens to be a particular case of a degree map in the sense of [Sai13, Section 4]. Degree maps are employed in [Sai13] to define a growth function and the skew-growth function for the monoid, which are then shown to be inverse to each other as Dirichlet series. In the notation of [Sai13] with a change of variable $t \mapsto e^{-\beta}$, we get

$$
P_{\operatorname{deg}}(\beta)=\sum_{[s] \in S / \sim} N_{s}^{-\beta}=\sum_{n \in N(S)} n^{1-\beta}=\zeta_{S}(\beta),
$$

the $\zeta$-function appearing in [ABLS17, Definition 4.2]. The skew-growth function appears in [ABLS17, Remark 7.4] and is crucial for the reconstruction formula for $\mathrm{KMS}_{\beta}$-states on $C^{*}(S)$ in [ABLS17, Lemma 7.5]. With this perspective and the inversion formula established in [Sai13], degree maps (with extra properties related to the intersection of 
right ideals) may yield interesting dynamics on $C^{*}$-algebras associated to cancellative monoids, for which the structure of KMS-states can be studied to a satisfactory degree. The assumption $S_{c}=S^{*}$ from above is needed to harmonize the two approaches as Saito identifies elements in the monoid up to $S^{*}$, while an identification up to $S_{c}$ is more natural in the approach pursued in [ABLS17]. It may thus be very interesting to see how much of Saito's theory can be transferred from $S^{*}$ to $S_{c}$.

\section{REFERENCES}

[ABLS17] Zahra Afsar, Nathan Brownlowe, Nadia S. Larsen, and Nicolai Stammeier, Equilibrium states on right LCM semigroup $C^{*}$-algebras, Int. Math. Res. Not., posted on 2017 (advance article), DOI 10.1093/imrn/rnx162.

[AR97] Victor Arzumanian and Jean Renault, Examples of pseudogroups and their $C^{*}$-algebras, Operator algebras and quantum field theory (Rome, 1996), 1997, pp. 93-104.

[BaHLR12] Nathan Brownlowe, Astrid an Huef, Marcelo Laca, and Iain Raeburn, Boundary quotients of the Toeplitz algebra of the affine semigroup over the natural numbers, Ergodic Theory Dynam. Systems 32 (2012), no. 1, 35-62, DOI 10.1017/S0143385710000830.

[BLRSt] Nathan Brownlowe, Nadia S. Larsen, Jacqui Ramagge, and Nicolai Stammeier, $C^{*}$-algebras of right LCM monoids and their equilibrium states. preprint, arXiv:1902.02674.

[BLS18] Nathan Brownlowe, Nadia Larsen, and Nicolai Stammeier, $C^{*}$-Algebras of algebraic dynamical systems and right LCM semigroups, Indiana Univ. Math. J. 67 (2018), no. 6, 2453-2486.

[BRRW14] Nathan Brownlowe, Jacqui Ramagge, David Robertson, and Michael F. Whittaker, ZappaSzép products of semigroups and their $C^{*}$-algebras, J. Funct. Anal. 266 (2014), no. 6, 3937-3967, DOI 10.1016/j.jfa.2013.12.025.

[BS16] Nathan Brownlowe and Nicolai Stammeier, The boundary quotient for algebraic dynamical systems, J. Math. Anal. Appl. 438 (2016), no. 2, 772-789, DOI 10.1016/j.jmaa.2016.02.015.

[BLRS19] Chris Bruce, Marcelo Laca, Jacqui Ramagge, and Aidan Sims, Equilibrium states and growth of quasi-lattice ordered monoids, to appear in Proc. Amer. Math. Soc., posted on 2019, DOI 10.1090/proc/14108.

[CF69] P. Cartier and D. Foata, Problèmes combinatoires de commutation et réarrangements, Lecture Notes in Mathematics, No. 85, Springer-Verlag, Berlin-New York, 1969.

[CaHR16] Lisa Orloff Clark, Astrid an Huef, and Iain Raeburn, Phase transition on the Toeplitz algebra of Baumslag-Solitar semigroups, Indiana Univ. Math. J. 65 (2016), 2137-2173, DOI 10.1512/iumj.2016.65.5934.

[VdC01] António Veloso da Costa, Graph products of monoids, Semigroup Forum 63 (2001), no. 2, 247-277, DOI 10.1007/s002330010075.

[CL02] John Crisp and Marcelo Laca, On the Toeplitz algebras of right-angled and finite-type Artin groups, J. Aust. Math. Soc. 72 (2002), no. 2, 223-245, DOI 10.1017/S1446788700003876.

[CL07] , Boundary quotients and ideals of Toeplitz $C^{*}$-algebras of Artin groups, J. Funct. Anal. 242 (2007), no. 1, 127-156, DOI 10.1016/j.jfa.2006.08.001.

[CV13] Joachim Cuntz and Anatoly Vershik, $C^{*}$-algebras associated with endomorphisms and polymorphisms of compact abelian groups, Comm. Math. Phys. 321 (2013), no. 1, 157-179, DOI 10.1007/s00220-012-1647-0.

[DR95] V. Diekert and G. Rozenberg (eds.), The book of traces, World Scientific Publishing Co., Inc., River Edge, NJ, 1995.

[ELR16] Søren Eilers, Xin Li, and Efren Ruiz, The isomorphism problem for semigroup $C^{*}$-algebras of right-angled Artin monoids, Doc. Math. 21 (2016), 309-343. Documenta Vol-21/11.

[ER07] Ruy Exel and Jean Renault, Semigroups of local homeomorphisms and interaction groups, Ergodic Theory Dynam. Systems 27 (2007), no. 6, 1737-1771, DOI $10.1017 / \mathrm{S} 0143385707000193$. 
[FK09] John Fountain and Mark Kambites, Graph products of right cancellative monoids, J. Aust. Math. Soc. 87 (2009), no. 2, 227-252, DOI 10.1017/S144678870900010X.

[Gre90] Elisabeth R. Green, Graph products of groups, University of Leeds, 1990.

[LR10] Marcelo Laca and Iain Raeburn, Phase transition on the Toeplitz algebra of the affine semigroup over the natural numbers, Adv. Math. 225 (2010), no. 2, 643-688, DOI 10.1016/j.aim.2010.03.007.

[LRR11] Marcelo Laca, Iain Raeburn, and Jacqui Ramagge, Phase transition on Exel crossed products associated to dilation matrices, J. Funct. Anal. 261 (2011), no. 12, 3633-3664, DOI 10.1016/j.jfa.2011.08.015.

[LRRW14] Marcelo Laca, Iain Raeburn, Jacqui Ramagge, and Michael F. Whittaker, Equilibrium states on the Cunt-Pimsner algebras of self-similar actions, J. Funct. Anal. 266 (2014), no. 11, 6619-6661, DOI 10.1016/j.jfa.2014.03.003.

[Led78] François Ledrappier, Un champ markovien peut être d'entropie nulle et mélangeant, C. R. Acad. Sci. Paris Sér. A-B 287 (1978), no. 7, A561-A563.

[Sai13] Kyoji Saito, Inversion formula for the growth function of a cancellative monoid, J. Algebra 385 (2013), 314-332, DOI 10.1016/j.jalgebra.2013.01.037.

[Spi12] Jack Spielberg, $C^{*}$-algebras for categories of paths associated to the Baumslag-Solitar groups, J. Lond. Math. Soc. (2) 86 (2012), no. 3, 728-754, DOI 10.1112/jlms/jds025.

[Sta15] Nicolai Stammeier, On $C^{*}$-algebras of irreversible algebraic dynamical systems, J. Funct. Anal. 269 (2015), no. 4, 1136-1179, DOI 10.1016/j.jfa.2015.02.005.

[Sta18] Graph products and the absence of property (AR), J. Aust. Math. Soc. 104 (2018), no. 2, 274-288, DOI 10.1017/S1446788717000192.

[Sta] _ Topological freeness for *-commuting covering maps, Houston J. Math., 1-27. to appear, arxiv:1311.0793.

[Star15] Charles Starling, Boundary quotients of $C^{*}$-algebras of right LCM semigroups, J. Funct. Anal. 268 (2015), no. 11, 3326-3356, DOI 10.1016/j.jfa.2015.01.001.

[SY10] Aidan Sims and Trent Yeend, $C^{*}$-algebras associated to product systems of Hilbert bimodules, J. Operator Theory 64 (2010), no. 2, 349-376.

Department of Mathematics, University of Oslo, P.O. Box 1053, Blindern, NO-0316 OSLO, NoRWAY

E-mail address: nicolsta@math.uio.no 